\title{
BMJ Open Does integrated care reduce hospital activity for patients with chronic diseases? An umbrella review of systematic reviews
}

\author{
Sarah Damery, Sarah Flanagan, Gill Combes
}

To cite: Damery $\mathrm{S}$,

Flanagan S, Combes G. Does integrated care reduce hospital activity for patients with chronic diseases? An umbrella review of systematic reviews. BMJ Open 2016;6: e011952. doi:10.1136/ bmjopen-2016-011952

- Prepublication history and additional material is available. To view please visit the journal (http://dx.doi.org/ 10.1136/bmjopen-2016011952).

Received 20 March 2016 Revised 8 August 2016 Accepted 30 September 2016

CrossMark

Institute of Applied Health Research, College of Medical and Dental Sciences, University of Birmingham, Edgbaston, UK

Correspondence to Dr Sarah Damery; s.I. damery@bham.ac.uk

\section{ABSTRACT}

Objective: To summarise the evidence regarding the effectiveness of integrated care interventions in reducing hospital activity.

Design: Umbrella review of systematic reviews and meta-analyses.

Setting: Interventions must have delivered care crossing the boundary between at least two health and/ or social care settings.

Participants: Adult patients with one or more chronic diseases.

Data sources: MEDLINE, Embase, ASSIA, PsycINFO, HMIC, CINAHL, Cochrane Library (HTA database, DARE, Cochrane Database of Systematic Reviews), EPPI-Centre, TRIP, HEED, manual screening of references.

Outcome measures: Any measure of hospital admission or readmission, length of stay (LoS), accident and emergency use, healthcare costs.

Results: 50 reviews were included. Interventions focused on case management $(n=8)$, chronic care model (CCM) $(n=9)$, discharge management $(n=15)$, complex interventions $(n=3)$, multidisciplinary teams (MDT) $(n=10)$ and self-management $(n=5) .29$ reviews reported statistically significant improvements in at least one outcome. 11/21 reviews reported significantly reduced emergency admissions (15-50\%); 11/24 showed significant reductions in all-cause $(10-30 \%)$ or condition-specific (15-50\%) readmissions; 9/16 reported LoS reductions of 1-7 days and 4/9 showed significantly lower A\&E use (30-40\%). 10/25 reviews reported significant cost reductions but provided little robust evidence. Effective interventions included discharge management with postdischarge support, MDT care with teams that include condition-specific expertise, specialist nurses and/or pharmacists and self-management as an adjunct to broader interventions. Interventions were most effective when targeting single conditions such as heart failure, and when care was provided in patients' homes.

Conclusions: Although all outcomes showed some significant reductions, and a number of potentially effective interventions were found, interventions rarely demonstrated unequivocally positive effects. Despite the centrality of integrated care to current policy, questions remain about whether the magnitude of

\section{Strengths and limitations of this study}

- This umbrella review is the first of its kind since integrated care became central to healthcare policy.

- Outcomes were selected following consultation with service providers, commissioners and patient representatives to ensure relevance.

- We assessed a large volume of international evidence across diverse chronic conditions, interventions and outcomes.

- Umbrella reviews do not allow conclusions to be drawn about the detailed contexts in which interventions were implemented, but they do permit a broader overview of the evidence base than would be possible with a focus on primary research alone.

- Heterogeneity of intervention design, duration, intensity and follow-up prohibited meta-synthesis across reviews.

potentially achievable gains is enough to satisfy national targets for reductions in hospital activity. Trial registration number: CRD42015016458.

\section{INTRODUCTION}

Hospital activity continues to rise and currently accounts for almost half of annual NHS expenditure. ${ }^{1}$ Demands on the acute sector are strongly influenced by the rapidly growing number of patients with multiple, chronic health conditions. These patients often need to access multiple health and social care settings but typically experience fragmented and poorly coordinated care. ${ }^{2} 3$ Reducing hospital activity is seen as the key to relieving pressure on services that are rapidly approaching their limits, ${ }^{4}$ and integrated care has become a cornerstone of the policy response to this challenge in the UK and most other developed countries. Integrated care represents an organising principle for care delivery that aims to 
improve patient experience of services through improved coordination across and between settings. ${ }^{5}$ By facilitating more patient contact, treatment and follow-up in primary care, in the community or in patients' homes, integration aims to reduce substantially the number of emergency and other admissions to hospital and facilitate timely and effective discharge from hospital to other settings. Following the establishment of a series of integrated care 'pioneers' in 2013, hospital trusts and commissioning organisations in England are planning and investing in a plethora of integrated services via the Better Care Fund (BCF), which aims to promote joint working at a strategic and operational level. ${ }^{6}$ Following the NHS Five Year Forward View, ${ }^{7}$ there are also proposals to develop and implement new models of care with integration as their central principle. $^{8}$

Integration undoubtedly has laudable aims-poor care coordination is often the main problem cited by patients when describing their experiences of health and social care services. ${ }^{9}{ }^{10}$ NHS staff also welcome integration, ${ }^{11} 12$ yet evidence about the effectiveness of integrated care in reducing healthcare resource use, particularly within the acute sector, is limited. Integrated care programmes can have a positive effect on service quality, ${ }^{13}$ and there is emerging evidence from recent evaluations of integrated care pilots that suggests potential for service efficiencies. ${ }^{14} 15$ However, there is still uncertainty about which interventions are most effective and how these should be implemented, ${ }^{16}$ alongside persistent questions over whether the aims of integration are ultimately achievable in any meaningful way. ${ }^{17}$ Given this uncertainty, it is timely to assess the evidence. This paper reports the findings of an umbrella review of the evidence for integrated care interventions operating across health and/or social care settings for chronic disease management in order to assess: (1) whether integration reduces hospital activity, (2) which interventions are the most promising, for which patients and in which settings, and (3) what are the associated cost implications.

\section{METHODS}

Umbrella reviews synthesise evidence from multiple systematic reviews into a single 'meta review', using the findings and conclusions of included systematic reviews as the raw data. They are useful when the evidence base is broad and are of particular importance for decision makers who need a synthesis of the most current and reliable data relevant to their context. ${ }^{18}$ The protocol was published ${ }^{19}$ and registered on PROSPERO.

\section{Inclusion criteria}

We included systematic reviews and meta-analyses published since January 2000 that evaluated interventions designed to facilitate integrated health and/or social care services. The year 2000 was chosen following scoping searches that indicated little or no systematic review evidence for integrated care interventions before this date. Eligible reviews could include primary studies of any experimental or quasi-experimental study design, providing the authors had identified studies using systematic methods. Eligibility was limited to reviews available in English.

Participants included adult patients with one or more chronic conditions. A list of 11 specific conditions was derived following a scoping review and combined a series of conditions recommended as central to any systematic review of chronic disease $\mathrm{e}^{20} 21$ and those included in the most recent Health Survey for England. ${ }^{22}$ The resulting conditions (hypertension, depression, diabetes, coronary heart disease, stroke, transient ischaemic attack, chronic obstructive pulmonary disease (COPD), cancer, heart failure, dementia and arthritis) covered those that are most prevalent within the adult population, most costly to manage and most likely to occur in combination with other chronic conditions.

Interventions could be implemented in any health or social care setting (primary, secondary or community care), as long as they crossed the boundary between two or more settings. The community setting encompassed care given in the community, in patient homes or by social care professionals. Exclusion criteria were: palliative care interventions; purely psychosocial interventions or those related to spirituality, mindfulness, health literacy or the use of complementary and alternative medicines; interventions focusing solely on diet and lifestyle factors; treatment or medication adherence; the effectiveness of surgical or diagnostic techniques; caregivers; pregnancy, and interventions implemented in less economically developed countries.

Comparison groups could include usual care, no intervention or comparison to one or more other interventions.

\section{Outcome measures}

Outcome measures were selected following a scoping review, a stakeholder workshop attended by service providers and commissioners and consultation with a group of patient and public involvement (PPI) advisors. Eligible reviews assessed one or more of the following outcomes: acute sector activity (emergency hospital admissions/readmissions, length of hospital stay, accident and emergency (A\&E) use) and healthcare costs.

\section{Search strategy}

The search strategy was intentionally broad and included general terms related to chronic disease, multimorbidities and long-term conditions as well as $\mathrm{MeSH}$ terms for the 11 specific chronic diseases identified from scoping searches. Search terms associated with integrated care and known interventions were also included. A separate search identified systematic reviews that assessed the cost implications of integrated care interventions (see online 
supplementary information for MEDLINE search strategy).

Relevant reviews were identified by searching electronic bibliographic databases and the manual checking of each included review's reference list. We searched MEDLINE, Embase, ASSIA (Applied Social Sciences Index and Abstracts), PsycINFO, Health Management Information Consortium database (HMIC), CINAHL, Cochrane library (including the Health Technology Assessment (HTA) database, Cochrane Database of Systematic Reviews, Database of Abstracts of Reviews of Effectiveness-DARE), EPPI-Centre library, TRIP database and the Health Economics Evaluations Database (HEED). Searches were performed in July 2014 and updated in December 2015.

\section{Eligibility assessment and data extraction}

Two authors (SD and SF) independently screened titles and abstracts against the inclusion and exclusion criteria, and full text copies of all potentially relevant reviews were assessed. Disagreements were resolved through the independent assessment of a third author (GC). Where multiple versions of an eligible review were available, the most recent or most comprehensive version was included. Where the same review was published more than once (eg, Cochrane Collaboration review and subsequent update), the updated version was included. Data on review characteristics (databases searched, geographical scope, healthcare settings and disease(s) focused on), methodology (aim, research questions, number of studies included, review type), study participants, interventions and outcomes of interest were extracted from each included review and crosschecked by SD and SF according to a predefined data extraction sheet. For narrative reviews, a statement summarising the authors' primary interpretation of findings was extracted. For meta-analyses, data on relative risks or
ORs were extracted along with the corresponding $95 \%$ CIs.

\section{Quality assessment}

Review quality was appraised independently by SD and SF using the Centre for Evidence-Based Medicine (CEBM) tool for critical appraisal of systematic reviews (http://www.cebm.net/wp-content/uploads/2014/04/SR Appraisal_sheet_2005_English.doc) which scores each review between 0 (poor quality) and 5 (high quality). Using quality score as an exclusion criterion was not part of the protocol, but considerable quality differences were evident between reviews scoring 0 to 2.5 and those scoring 3 or above. Lower quality reviews had little (if any) extractable outcomes data so we decided to exclude reviews scoring 2.5 or less on the quality scale.

\section{Data analysis}

Heterogeneity in study populations, interventions assessed, follow-up periods and specification of control groups prevented pooling of intervention effects and quantitative meta-synthesis across reviews. Owing to this, and to avoid the risk of 'double counting' evidence where multiple reviews contained some of the same primary studies, our synthesis was a primarily narrative review of interventions and outcomes. The strength of evidence from each meta-analysis or narrative review was characterised according to four categories: definite positive or negative associations, mixed findings or no association (table 1).

\section{RESULTS}

Figure 1 summarises the search. A total of 11436 potentially eligible reviews were identified, and 50 (in 49 papers) were included (table 2). A total of 1208 individual primary studies were included in the reviews (median 19, range 4-153). Nineteen reviews did not specify patient numbers, but across the 31 that did, all

\section{Table 1 Categorisation of the strength of effect for included reviews}

\begin{tabular}{|c|c|c|}
\hline Category & Symbol & Interpretation \\
\hline $\begin{array}{l}\text { Positive } \\
\text { association }\end{array}$ & + & $\begin{array}{l}\text { At least half of a review's included primary studies showed a statistically significant increase in } \\
\text { a particular outcome following the intervention; the authors' summary of findings (narrative } \\
\text { reviews) demonstrated a positive association, or pooled results from a meta-analysis indicated } \\
\text { a statistically significant positive association, eg, hospital admission rates significantly } \\
\text { increased. }\end{array}$ \\
\hline $\begin{array}{l}\text { Negative } \\
\text { association }\end{array}$ & - & $\begin{array}{l}\text { At least half of a review's included primary studies showed a statistically significant decrease in } \\
\text { a particular outcome following the intervention; the authors' summary of findings (narrative } \\
\text { reviews) demonstrated a negative association, or pooled results from a meta-analysis indicated } \\
\text { a statistically significant negative association, eg, hospital admission rates significantly } \\
\text { reduced. }\end{array}$ \\
\hline Mixed findings & $?$ & $\begin{array}{l}\text { A review reported mixed findings, in which some primary studies may have shown a } \\
\text { statistically significant difference between intervention and control groups whereas other } \\
\text { primary studies showed no significant differences between groups. }\end{array}$ \\
\hline No association & $=$ & $\begin{array}{l}\text { A review where no significant differences between intervention and control groups were } \\
\text { reported in any of the included primary studies. }\end{array}$ \\
\hline
\end{tabular}


Figure 1 PRISMA diagram of search results.

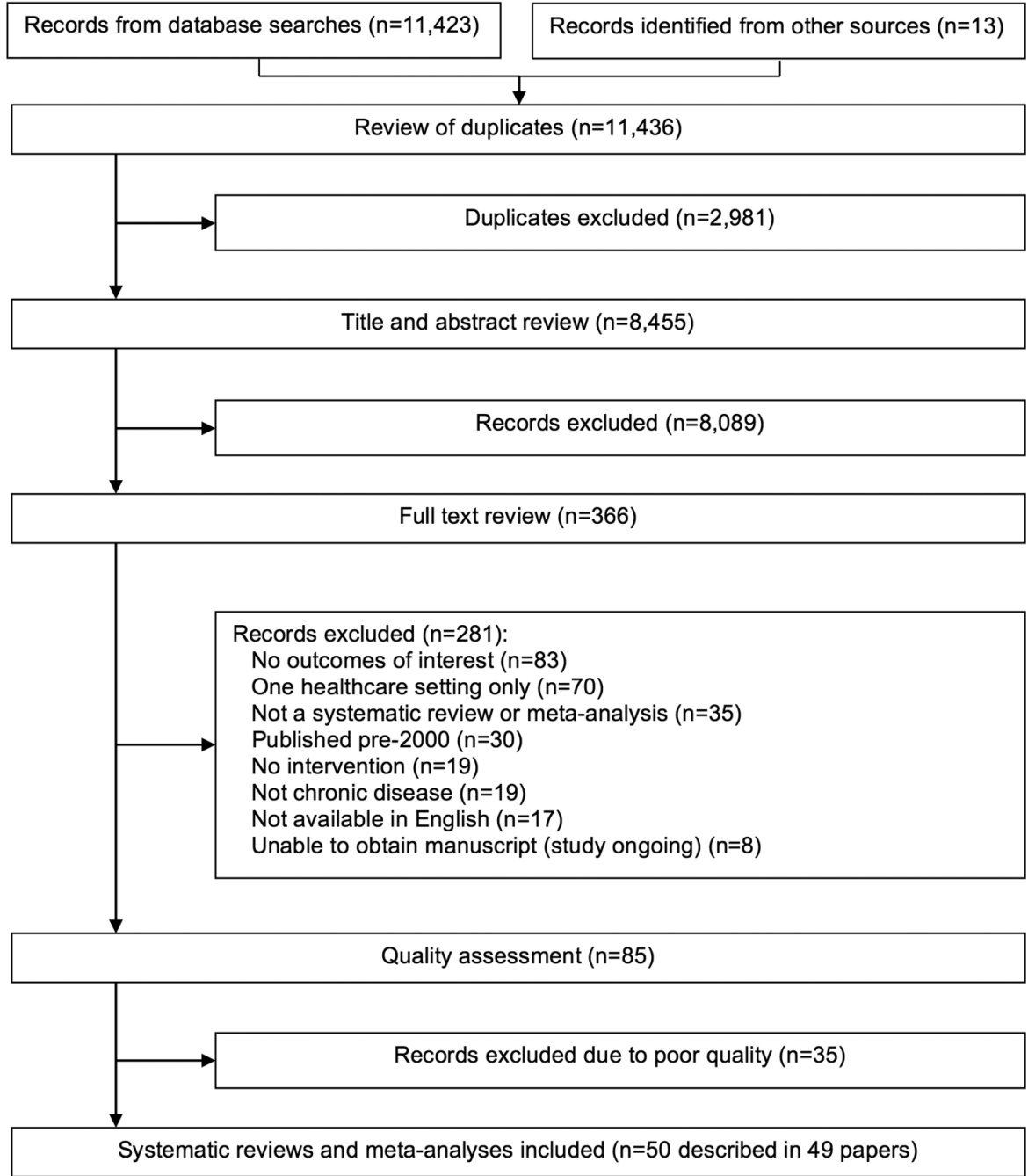

but three included 1000 or more patients (total 219475 , median 2692, range 857-78 590). Studies within reviews varied in duration from 2 weeks to 60 months, with most lasting up to 12 months, although 9 reviews did not specify follow-up duration. Furthermore, 21 reviews were narrative, ${ }^{23-42} 26$ included meta-analyses ${ }^{43-68}$ and 3 were reviews of reviews. ${ }^{69-71}$

The most commonly studied condition was chronic disease $(\mathrm{n}=15),{ }^{23-28} 37 \quad 39-4143576970$ followed by heart failure $(\mathrm{n}=14),{ }^{36} \quad 42 \quad 45 \quad 47 \quad 53 \quad 55 \quad 58 \quad 59 \quad 61-66$ COPD

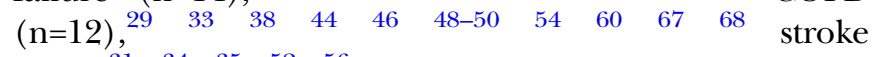
$(\mathrm{n}=5), 31 \quad 34 \quad 35 \quad 52 \quad 56$ stroke and cardiac conditions $(\mathrm{n}=2),{ }^{30}$ mental health $(\mathrm{n}=1)^{51}$ and heart failure and COPD combined $(\mathrm{n}=1){ }^{71}$ All reviews were published between 2004 and 2015. Reviews were published in Canada, ${ }^{26} 31 \quad 37 \quad 3841 \quad 42 \quad 57 \quad 63 \quad 65-67 \quad 71$ the UK, ${ }^{24}$ 43-45 $525660-6264$ the USA, ${ }^{23} 3032334651535859$ the Netherlands, $^{25} \quad{ }^{27-29} \quad 34 \quad 48 \quad 49 \quad 68$ Ireland, ${ }^{39} \quad 40$ Switzerland, ${ }^{50} 70$ Norway, ${ }^{54}$ Japan, ${ }^{69}$ Hong Kong, ${ }^{36}$ Spain, ${ }^{47}$ Denmark ${ }^{35}$ and Greece. ${ }^{55}$ In most reviews, the comparator was usual clinical care, although a detailed description of usual care was typically not provided. Overall, 29 reviews $(58 \%)$ reported a nominally statistically significant result for at least one outcome.

\section{Quality of included reviews}

The mean quality assessment (QA) score was $4 / 5$.

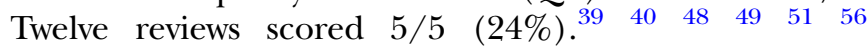
5861626668 The criterion for which the largest number of reviews failed to score a point related to whether a valid consideration of bias across primary studies had been undertaken. There was no discernible trend in review quality across intervention categories: the mean QA scores by the intervention group ranged from 3.4/5 (case management) to $4.2 / 5$ (chronic care model (CCM), multidisciplinary teams (MDT), selfmanagement).

\section{Effects by intervention type}

Interventions were categorised into six broad groups (table 3), although intervention components frequently overlapped.

Eight reviews focused on case management interventions. ${ }^{23-27}{ }^{43-45}$ With the exception of one review which showed that case management was associated with significantly reduced healthcare costs, ${ }^{26}$ and another that demonstrated a $49 \%$ relative risk reduction (RRR) in admissions for patients with heart failure, ${ }^{45}$ all case management reviews showed mixed findings or no 


\begin{tabular}{|c|c|c|c|c|c|}
\hline Author (year); country & $\begin{array}{l}\text { Study types, } n \text {; } \\
\text { participants } n \text {; } \\
\text { databases }\end{array}$ & $\begin{array}{l}\text { Condition(s); } \\
\text { population(s); } \\
\text { review type }\end{array}$ & $\begin{array}{l}\text { Comparator; QA } \\
\text { score; time scale }\end{array}$ & $\begin{array}{l}\text { Intervention summary; health/ } \\
\text { social care settings }\end{array}$ & $\begin{array}{l}\text { Findings of review by outcome } \\
\text { (intervention vs control) }\end{array}$ \\
\hline \multicolumn{6}{|l|}{ Case management } \\
\hline $\begin{array}{l}\text { Hickam et al (2013); } \\
\text { USA }\end{array}$ & $\begin{array}{l}\text { RCTs, } \\
\text { observational } \\
n=153 \\
\text { Not specified } \\
5 \text { databases, } \\
\text { inception-2011 }\end{array}$ & $\begin{array}{l}\text { Chronic disease } \\
\text { Adult patients with } \\
\text { complex care } \\
\text { needs } \\
\text { Narrative }\end{array}$ & $\begin{array}{l}\text { Care without a case } \\
\text { management } \\
\text { component } \\
4 \\
6-36 \text { months }\end{array}$ & $\begin{array}{l}\text { Intensive interventions: multiple } \\
\text { face-to-face interactions, home } \\
\text { visits vs less intensive } \\
\text { interventions: infrequent contact } \\
\text { Delivered by case managers } \\
\text { working alone or within a MDT of } \\
\text { health professionals. } \\
\text { Primary, secondary, community }\end{array}$ & $\begin{array}{l}\text { Admissions: } 2 \text { studies found case } \\
\text { management to be more effective } \\
\text { in patients with greater disease } \\
\text { burden. A further } 4 \text { studies found } \\
\text { case management to be effective } \\
\text { when case managers have greater } \\
\text { levels of personal contact with } \\
\text { patients (low quality evidence). } \\
\text { Costs ( } 4 \text { studies): } 3 \text { found no } \\
\text { difference between groups. One } \\
\text { study found higher overall costs in } \\
\text { intervention group vs control. }\end{array}$ \\
\hline Hutt et al (2004); ${ }^{24}$ UK & $\begin{array}{l}\text { RCTs, CCTs, } \\
\text { before/after } n=20 \\
n=18002 \\
\text { 'Major' databases, } \\
1996-2004\end{array}$ & $\begin{array}{l}\text { Chronic disease } \\
\text { Over } 65 \text { s with any } \\
\text { chronic condition } \\
\text { (mental health } \\
\text { excluded) } \\
\text { Narrative }\end{array}$ & $\begin{array}{l}\text { Care without a case } \\
\text { management } \\
\text { component } \\
3 \\
\text { At least } 3 \text { months }\end{array}$ & $\begin{array}{l}\text { Home visits and/or periodic } \\
\text { reassessment, ranging from case } \\
\text { manager assessment at hospital or } \\
\text { home with occasional telephone } \\
\text { contact, to regular intensive contact } \\
\text { where case managers arranged } \\
\text { medical appointments and were } \\
\text { contactable } 24 / 7 \text {. } \\
\text { Delivered by case manager (nurse } \\
\text { or social worker). } \\
\text { Primary, secondary, community. }\end{array}$ & $\begin{array}{l}\text { Admissions (18 studies): } 5 \text { showed } \\
\text { a significant reduction in } \\
\text { admissions in intervention groups; } \\
7 \text { found no difference between } \\
\text { groups; } 4 \text { found non-significant } \\
\text { reductions, } 2 \text { found non-significant } \\
\text { increases in admissions in the } \\
\text { intervention group } \\
\text { LoS (16 studies): } 3 / 16 \text { showed } \\
\text { significant decrease, } 2 / 16 \text { showed } \\
\text { non-significant increase, } 11 / 16 \\
\text { showed no differences. } \\
\text { A\&E use ( } 8 \text { studies): } 3 \text { showed } \\
\text { significant reduction, } 2 \text { showed } \\
\text { significant increases, } 2 \text { showed } \\
\text { non-significant increases. } \\
\text { Costs (10 studies): } 4 \text { showed } \\
\text { non-significant increases in } \\
\text { intervention; } 6 \text { reported reductions } \\
\text { although only } 1 \text { was significant. }\end{array}$ \\
\hline $\begin{array}{l}\text { Latour et al (2007); } \\
\text { Netherlands }\end{array}$ & $\begin{array}{l}\text { RCTs, CCTs, } \\
\text { before/after } n=10 \\
n=5092 \\
4 \text { databases, } \\
\text { inception- } 2005\end{array}$ & $\begin{array}{l}\text { Chronic disease } \\
\text { Adult patients with } \\
\text { acute or chronic } \\
\text { conditions } \\
\text { Narrative }\end{array}$ & $\begin{array}{l}\text { Care without a case } \\
\text { management } \\
\text { component } \\
3 \\
\text { 3-18 months }\end{array}$ & $\begin{array}{l}\text { Postdischarge nurse-led case } \\
\text { management for complex patients, } \\
\text { delivered in the outpatient setting. } \\
\text { Needs assessment, service plans, } \\
\text { monitoring, assessment, } \\
\text { evaluation, follow-up via home }\end{array}$ & $\begin{array}{l}\text { Readmissions ( } 9 \text { studies): } 3 \text { high } \\
\text { quality, } 1 \text { low quality reported } \\
\text { positive results for intervention. } \\
4 \text { studies ( } 2 \text { high quality) showed } \\
\text { no difference between groups; } \\
1 \text { presented insufficient data. }\end{array}$ \\
\hline
\end{tabular}




\begin{tabular}{|c|c|c|c|c|c|}
\hline Author (year); country & $\begin{array}{l}\text { Study types, } \mathrm{n} ; \\
\text { participants } n \text {; } \\
\text { databases }\end{array}$ & $\begin{array}{l}\text { Condition(s); } \\
\text { population(s); } \\
\text { review type }\end{array}$ & $\begin{array}{l}\text { Comparator; QA } \\
\text { score; time scale }\end{array}$ & $\begin{array}{l}\text { Intervention summary; health/ } \\
\text { social care settings }\end{array}$ & $\begin{array}{l}\text { Findings of review by outcome } \\
\text { (intervention vs control) }\end{array}$ \\
\hline & & & & $\begin{array}{l}\text { visits and/or telephone. } \\
\text { Secondary, community }\end{array}$ & $\begin{array}{l}\text { LoS ( } 6 \text { studies): } 2 \text { showed } \\
\text { significant reduction, } 2 \text { showed } \\
\text { non-significant reduction, } 2 \text { showed } \\
\text { no difference between groups. } \\
\text { A\&E use ( } 4 \text { studies): Strong } \\
\text { evidence that intervention had no } \\
\text { significant impact. }\end{array}$ \\
\hline $\begin{array}{l}\text { Manderson et al (2012); } \\
\text { Canada }\end{array}$ & $\begin{array}{l}\text { RCTs } n=15 \\
n=2317 \\
5 \text { databases, } \\
1999-2011\end{array}$ & $\begin{array}{l}\text { Chronic disease } \\
\text { Older people } \\
\text { Narrative }\end{array}$ & $\begin{array}{l}\text { Not specified } \\
3 \\
1-18 \text { months }\end{array}$ & $\begin{array}{l}\text { Care planning and coordination via } \\
\text { phone support, home visits, liaison } \\
\text { with medical and community } \\
\text { services and/or education. APN, } \\
\text { care coordinators, case managers. } \\
\text { Primary, secondary, community }\end{array}$ & $\begin{array}{l}\text { Costs ( } 9 \text { studies): } 5 \text { reported } \\
\text { positive economic outcomes, } 4 \text { did } \\
\text { not. Specific data and effect sizes } \\
\text { not given. }\end{array}$ \\
\hline $\begin{array}{l}\text { Oeseburg et al (2009); }{ }^{27} \\
\text { Netherlands }\end{array}$ & $\begin{array}{l}\text { RCTs } n=9 \\
n=15746 \\
3 \text { databases, } \\
1995-2007\end{array}$ & $\begin{array}{l}\text { Chronic disease } \\
\text { Community } \\
\text { dwelling patients } \\
\text { Narrative }\end{array}$ & $\begin{array}{l}\text { Care without a case } \\
\text { management } \\
\text { component } \\
3 \\
10-36 \text { months }\end{array}$ & $\begin{array}{l}\text { Home visits and/or telephone calls. } \\
\text { Delivered by a case manager } \\
\text { (nurse, social worker or nurse } \\
\text { practitioner) who was either a } \\
\text { member of a MDT or acted } \\
\text { independently. } \\
\text { Primary, secondary, community }\end{array}$ & $\begin{array}{l}\text { Admissions ( } 6 \text { studies): } 1 \text { showed } \\
\text { small reduction in favour of } \\
\text { intervention (good quality). One } \\
\text { found small increase in intervention } \\
\text { group (weak quality). } \\
\text { LoS ( } 5 \text { studies): One reported } \\
\text { small reduction in days per year in } \\
\text { hospital in intervention group. } \\
\text { A\&E use ( } 5 \text { studies): One reported } \\
\text { small reduction in intervention, } \\
1 \text { reported an increase. } 3 \text { reported } \\
\text { no difference. } \\
\text { Costs ( } 3 \text { studies): } 1 \text { reported } \\
\text { significant but trivial saving in } \\
\text { intervention. Another found } 19 \% \\
\text { cost reduction due to savings in } \\
\text { nursing home, hospital and } \\
\text { community costs. A third found } \\
\text { costs to be higher in intervention } \\
\text { (non-significant). }\end{array}$ \\
\hline Stokes et al (2015); ${ }^{43}$ UK & $\begin{array}{l}\text { RCTs, CCTs, } \\
\text { before/after, time } \\
\text { series } n=36 \\
n=23711 \\
6 \text { databases, } \\
\text { inception-2014 }\end{array}$ & $\begin{array}{l}\text { Chronic disease } \\
\text { Adult patients with } \\
\text { chronic diseases } \\
\text { SR and } \\
\text { meta-analysis }\end{array}$ & $\begin{array}{l}\text { Care without a case } \\
\text { management } \\
\text { component } \\
4 \\
6-60 \text { months }\end{array}$ & $\begin{array}{l}\text { Community-based MDTs } \\
\text { responsible for delivering and } \\
\text { coordinating services; MDT care } \\
\text { plan following case worker } \\
\text { assessment, case manager } \\
\text { constantly available to deal with } \\
\text { problems. }\end{array}$ & $\begin{array}{l}\text { Costs: No significant effects found: } \\
\text { Short term }(0-12 \text { months): SMD } \\
-0.00 \mathrm{Cl}-0 / 07 \text { to } 0.06 \\
\text { Longer term ( } 13+\text { months): SMD } \\
-0.03 \mathrm{Cl}-0.16 \text { to } 0.10\end{array}$ \\
\hline
\end{tabular}


Taylor et al (2005); $;^{44}$ UK

RCTs $\mathrm{n}=9$

$n=1428$

24 databases,

1980-2005

RCTs $\mathrm{n}=10$

Not specified

18 databases,

inception-2010
COPD

Patients with

COPD in the

community

SR and

meta-analysis

Heart failure

Adult patients

SR and

meta-analysis

Not specified

3

3-18 months

Conventional

postdischarge care

4.5

3-12 months

COPD

Not specified

RCTs $\mathrm{n}=32$

Not specified

3 databases,

inception-2005
6 weeks to 24 months
Adult patients with

COPD

$\mathrm{SR}$ and

meta-analysis score; timer; QA
Intervention summary; health/

social care settings

Delivered by care manager, nurse pharmacist, GP collaborating with nurse.

Primary, community

Brief (1 month) or longer term

(12 months) inpatient, outpatient or

community-based interventions. All were led, coordinated or delivered by respiratory nurses via home visits, with or without telephone follow-up.

Primary, secondary, community

Specialist HF management

education

1. Intensive: 4-6-week

appointments

2. Decreasing intensity: every 1-2 weeks for 3 months, then every 3 months

3. Regular: 3-4-month appointments

4. Tailored: appointments by patient need

5. Primary, secondary

At least one component of Wagner's CCM. Categorised according to the number of components an intervention included.

Primary, secondary, community
Findings of review by outcome (intervention vs control)

Readmissions: Equivocal evidence for reduction in all-cause readmission at 12 months. One study found a $40 \%$ reduction in readmission for acute exacerbation and $57 \%$ reduction in all-cause readmission. Another found a significant reduction in

readmissions. Three further studies found no effect.

Admissions:

At 3 months (RR $0.10,95 \% \mathrm{Cl}$ 0.01 to 0.78 ).

At 12 months (5 studies), $49 \%$ reduction in relative risk ( $\mathrm{RR} 0.51$, $95 \% \mathrm{Cl} 0.41$ to 0.63 )

At 18 months (1 study), no difference between groups. Interventions with decreasing intensity showed $58 \%$ reduction (RR $0.42,95 \% \mathrm{Cl} 0.27$ to 0.65 ). No significance for other groups.

Admissions: No difference in rates for interventions with $1 \mathrm{CCM}$ component $(n=7)$. Significant reduction for interventions with multiple CCM components $(n=4)$ RR $0.78,95 \% \mathrm{Cl} 0.66$ to 0.94 ) LoS:

1 CCM component (4 studies): No difference between groups.

Multiple components (2 studies):

Significant reduction in intervention (-2.51 days, $95 \% \mathrm{Cl}-3.40$ to $-1.61)$. 
Findings of review by outcome (intervention vs control)

A\&E use: 3 studies with 2+ CCM components found statistically significant reduction (RR $0.58,95 \%$ $\mathrm{Cl} 0.42$ to 0.79 ). Costs (7 studies): 3 RCTs showed $34 \%$ to $70 \%$ cost reduction with intervention. One RCT showed non-significant cost reductions. Three before/after studies reported an $11 \%$ to $23 \%$ reduction in costs after intervention.

de Bruin et al (2012); ${ }^{28}$

Netherlands

Gonseth et al (2004); 47

Spain

Hisashige (2013); ${ }^{69}$

Japan
RCTs, CCTs,

before/after,

case-control $\mathrm{n}=41$

$n=78590$

6 databases,

1995-2011

RCTs, CCTs,

$\mathrm{n}=27$

Not specified

3 databases,

inception-2003

Not specified

Adult patients with 4.5

Not specified

Narrative

Heart failure

Over 65s with

principle or

secondary

diagnosis of $\mathrm{HF}$

$\mathrm{SR}$ and

meta-analysis

SR and

meta-analyses

Chronic disease

Adult patients

$n=28$

component

4.5

3-48 months
Not specified

3.5

Not specified
Studies categorised by number of

CCM components they included.

Multiple settings, from home care organisations and community centres to primary care, hospitals, specialist clinics. Some included newly established partnerships; others provided regular care in settings where it was not normally given.

Primary, secondary, community

Care without a CCM

Education, counselling, diet ad

self-care support, discharge planning, focus on hospital to home transition, medication management, clinic review, GP follow-up.

Most delivered by nurses. Varied timing (eg, in-hospital or postdischarge), organisation (eg, home care or outpatient clinic visit), duration (from single home visit to intensive intervention lasting

12 months).

Primary, secondary, community

All interventions had 1+ CCM

component. Typically

multidisciplinary approaches with dmissions: $3 / 16$ studies found significantly reduced admissions. Costs (5 studies): All reported negative incremental direct healthcare costs for patients receiving intervention. Costs ranged from -US\$5708 to -US $\$ 204$ per patient per year, primarily due to lower inpatient costs in the intervention group.

Readmissions: Reduced regardless of follow-up length or whether intervention delivered at home or in clinic setting.

All-cause (6 studies): 15\% reduction in readmissions (RR $0.85,95 \% \mathrm{Cl} 0.79$ to 0.92 ). HF-specific (6 studies): $30 \%$ reduction in readmissions (RR $0.70,95 \% \mathrm{Cl} 0.62$ to 0.79 ) Costs (11 studies): 10 estimated the intervention reduced costs.

One reported similar costs in intervention and usual care groups. Admissions (22 studies):

'Improvement with a reasonable amount of evidence' with 


\begin{tabular}{|c|c|c|c|c|c|}
\hline Author (year); country & $\begin{array}{l}\text { Study types, } n ; \\
\text { participants } n ; \\
\text { databases }\end{array}$ & $\begin{array}{l}\text { Condition(s); } \\
\text { population(s); } \\
\text { review type }\end{array}$ & $\begin{array}{l}\text { Comparator; QA } \\
\text { score; time scale }\end{array}$ & $\begin{array}{l}\text { Intervention summary; health/ } \\
\text { social care settings }\end{array}$ & $\begin{array}{l}\text { Findings of review by outcome } \\
\text { (intervention vs control) }\end{array}$ \\
\hline & $\begin{array}{l}\text { Not specified } \\
9 \text { databases, } \\
1995-2010\end{array}$ & & & $\begin{array}{l}\text { clinical follow-up by specialists, } \\
\text { home visits, hospital discharge } \\
\text { planning or postdischarge } \\
\text { follow-up, counselling in hospital } \\
\text { and patient education or reminders. } \\
\text { Primary, secondary, community }\end{array}$ & $\begin{array}{l}\text { intervention seen in } 63 \% \text { of studies } \\
\text { (14/22). } \\
\text { Costs ( } 16 \text { studies): } 6 / 16 \text { (38\%) } \\
\text { observed 'improvement in costs } \\
\text { with a reasonable amount of } \\
\text { evidence'. Costs tended to focus } \\
\text { on healthcare costs and typically } \\
\text { did not include estimates of } \\
\text { intervention costs. }\end{array}$ \\
\hline $\begin{array}{l}\text { Kruis et al (2013); }{ }^{48} \\
\text { Netherlands }\end{array}$ & $\begin{array}{l}\text { RCTs } n=26 \\
n=2997 \\
5 \text { databases, } \\
1990 \text {-present }\end{array}$ & $\begin{array}{l}\text { COPD } \\
\text { Adult patients with } \\
\text { clinical diagnosis } \\
\text { of COPD } \\
\text { SR and } \\
\text { meta-analysis }\end{array}$ & $\begin{array}{l}\text { Regular follow-up visits } \\
\text { to healthcare providers } \\
5 \\
3-24 \text { months }\end{array}$ & $\begin{array}{l}\text { Multidisciplinary ( } 2+\text { providers), } \\
\text { multitreatment ( } 2+\mathrm{CCM} \\
\text { components), 3+ months duration. } \\
\text { Categorised as: } \\
\text { 1. Exercise dominant } \\
\text { 2. Self-management dominant } \\
\text { 3. Structured nurse/GP follow-up } \\
\text { 4. Exercise and self-management } \\
\text { 5. Self-management+structured } \\
\text { follow-up } \\
\text { 6. Individually tailored education } \\
\text { Primary, secondary, community }\end{array}$ & $\begin{array}{l}\text { Admissions: All-cause: number of } \\
\text { participants with one or more } \\
\text { admissions over } 3-12 \text { months was } \\
27 \text { per } 100 \text { in control vs } 20 \text { per } 100 \\
\text { in intervention (OR } 0.68,95 \% \mathrm{Cl} \\
0.47 \text { to } 0.99, \mathrm{p}=0.04) \text {. } \\
\text { Respiratory related: at } 3 \text { months } \\
\text { ( } 7 \text { studies), significant reduction } \\
(0.68,95 \% \mathrm{Cl} 0.47 \text { to } 0.99 \text {, } \\
\mathrm{p}=0.04) \text {. At } 12 \text { months ( } 1 \text { study), no } \\
\text { difference observed. } \\
\text { LoS: Significantly lower in the } \\
\text { intervention group. Mean difference } \\
-3.78 \text { days (95\% } \mathrm{Cl}-5.90 \text { to } \\
-1.67, \mathrm{p}<0.001) \text {. }\end{array}$ \\
\hline $\begin{array}{l}\text { Lemmens et al (2009); } \\
\text { Netherlands }\end{array}$ & $\begin{array}{l}\text { RCTs, before/after } \\
\mathrm{n}=36 \\
\text { Not specified } \\
2 \text { databases, } \\
1995-2008\end{array}$ & $\begin{array}{l}\text { COPD } \\
\text { Adult patients with } \\
\text { asthma or COPD } \\
\text { SR and } \\
\text { meta-analysis }\end{array}$ & $\begin{array}{l}\text { Care with } 0 \text { or } 1 \mathrm{CCM} \\
\text { components } \\
5 \\
6 \text { weeks to } 24 \text { months }\end{array}$ & $\begin{array}{l}\text { 1. Patient education+case } \\
\text { management } \\
\text { 2. Patient education+case } \\
\text { management+professional } \\
\text { education } \\
\text { 3. Patient education with } \\
\text { substitution of physician by } \\
\text { nurse } \\
\text { 4. Professional and patient } \\
\text { education combined with } \\
\text { pharmacists having an active } \\
\text { role in patient monitoring } \\
\text { Primary, secondary, community }\end{array}$ & $\begin{array}{l}\text { Readmissions: } \\
\text { Group } 1 \text { (6 studies): } 1 \text { showed } \\
\text { significant reduction } \\
\text { Group } 2 \text { ( } 6 \text { studies): } 3 \text { showed } \\
\text { significant reduction } \\
\text { Group } 3 \text { : No differences between } \\
\text { groups } \\
\text { Group } 4 \text { (8 studies): Ambiguous } \\
\text { results in all studies. }\end{array}$ \\
\hline
\end{tabular}




\begin{tabular}{|c|c|c|c|c|c|}
\hline Author (year); country & $\begin{array}{l}\text { Study types, } \mathrm{n} ; \\
\text { participants } n ; \\
\text { databases }\end{array}$ & $\begin{array}{l}\text { Condition(s); } \\
\text { population(s); } \\
\text { review type }\end{array}$ & $\begin{array}{l}\text { Comparator; QA } \\
\text { score; time scale }\end{array}$ & $\begin{array}{l}\text { Intervention summary; health/ } \\
\text { social care settings }\end{array}$ & $\begin{array}{l}\text { Findings of review by outcome } \\
\text { (intervention vs control) }\end{array}$ \\
\hline $\begin{array}{l}\text { Peytremann-Bridevaux } \\
\text { et al (2008); }{ }^{50} \\
\text { Switzerland }\end{array}$ & $\begin{array}{l}\text { RCTs, controlled } \\
\text { before/after } n=13 \\
n=8179 \\
5 \text { databases, } \\
\text { inception-2006 }\end{array}$ & $\begin{array}{l}\text { COPD } \\
\text { Adult patients } \\
\text { undergoing } \\
\text { disease } \\
\text { management } \\
\text { SR and } \\
\text { meta-analysis }\end{array}$ & $\begin{array}{l}\text { Care without a CCM } \\
\text { component } \\
4 \\
12 \text { months }\end{array}$ & $\begin{array}{l}\text { All included } 2+\text { CCM components; } \\
\text { at least } 1 \text { component must have } \\
\text { lasted } 12 \text { months. } \\
\text { Delivered by } 2+\text { health } \\
\text { professionals, eg, respiratory } \\
\text { nurse, physiotherapist, GP, practice } \\
\text { nurse, social worker, case } \\
\text { manager, pulmonary care } \\
\text { physician. } \\
\text { Primary, secondary, community }\end{array}$ & $\begin{array}{l}\text { Admissions ( } 10 \text { studies): } 7 \text { showed } \\
\text { significant effects in favour of } \\
\text { intervention; } 3 \text { found no reduction } \\
\text { in admissions. }\end{array}$ \\
\hline $\begin{array}{l}\text { Steuten et al (2009); } \\
\text { Netherlands }\end{array}$ & $\begin{array}{l}\text { Any with data at } \\
\text { two time points } \\
n=20 \\
\text { Not specified } \\
2 \text { databases, } \\
2005-2007\end{array}$ & $\begin{array}{l}\text { COPD } \\
\text { Mild, moderate, } \\
\text { severe or very } \\
\text { severe COPD } \\
\text { Narrative }\end{array}$ & $\begin{array}{l}\text { Care without a CCM } \\
\text { component } \\
3.5 \\
2-24 \text { months }\end{array}$ & $\begin{array}{l}\text { All included } 2+\text { CCM components. } \\
\text { All included self-management and } \\
\text { delivery system redesign. Several } \\
\text { programmes additionally } \\
\text { encompassed decision support } \\
\text { and/or clinical information systems } \\
\text { Primary, secondary, community }\end{array}$ & $\begin{array}{l}\text { Readmissions: } 8 / 15 \text { studies } \\
\text { reported a reduction in readmission } \\
\text { rates (3 statistically significant). } \\
\text { Relative risk of readmission ranged } \\
\text { from } 0.64 \text { to } 1.50 \text {. Statistically } \\
\text { significant improvements all seen } \\
\text { in studies with } 3 \text { or } 4 \text { intervention } \\
\text { components. Studies with fewer } \\
\text { components showed no significant } \\
\text { reductions. } \\
\text { Costs ( } 3 \text { studies): Differences } \\
\text { found in individual domains, eg, } \\
\text { higher prescription costs, lower } \\
\text { hospital costs, reduced sick leave } \\
\text { costs. No studies reported } \\
\text { statistically significant findings. }\end{array}$ \\
\hline $\begin{array}{l}\text { Woltmann et al (2012); } \\
\text { USA }\end{array}$ & $\begin{array}{l}\text { RCTs } \mathrm{n}=78 \\
\text { Not specified } \\
6 \text { databases, } \\
\text { inception-2011 }\end{array}$ & $\begin{array}{l}\text { Mental health } \\
\text { Adult patients with } \\
\text { mental health } \\
\text { problems } \\
\text { SR and } \\
\text { meta-analysis }\end{array}$ & $\begin{array}{l}\text { Not specified } \\
5 \\
3-36 \text { months }\end{array}$ & $\begin{array}{l}\text { Eligible interventions had to have } \\
\text { at least } 3 \text { CCM components. } \\
\text { Primary, secondary, community }\end{array}$ & $\begin{array}{l}\text { Costs ( } 21 \text { studies): } 10 \text { reported p } \\
\text { values. } 9 \text { of these reported no } \\
\text { difference between intervention } \\
\text { and control groups; } 1 \text { favoured } \\
\text { control condition. No statistically } \\
\text { significant findings in any study. }\end{array}$ \\
\hline $\begin{array}{l}\text { Discharge management } \\
\text { Bettger et al (2012); } \\
\text { USA }\end{array}$ & $\begin{array}{l}\text { RCTs, } \\
\text { observational, } \\
\mathrm{n}=44 \\
\text { Not specified }\end{array}$ & $\begin{array}{l}\text { Stroke, Cardiac } \\
\text { Patients } \\
\text { hospitalised for } \\
\text { stroke/MI } \\
\text { Narrative }\end{array}$ & $\begin{array}{l}\text { Not specified } \\
4 \\
\text { Not specified }\end{array}$ & $\begin{array}{l}\text { 1. Hospital-initiated discharge } \\
\text { support } \\
\text { 2. Community-based support } \\
\text { models }\end{array}$ & $\begin{array}{l}\text { Readmissions: } \\
\text { Hospital-initiated support: No } \\
\text { impact on readmission rates in } 6 \\
\text { studies focusing on stroke; no } \\
\text { impact in } 3 \text { studies focusing on MI }\end{array}$ \\
\hline
\end{tabular}




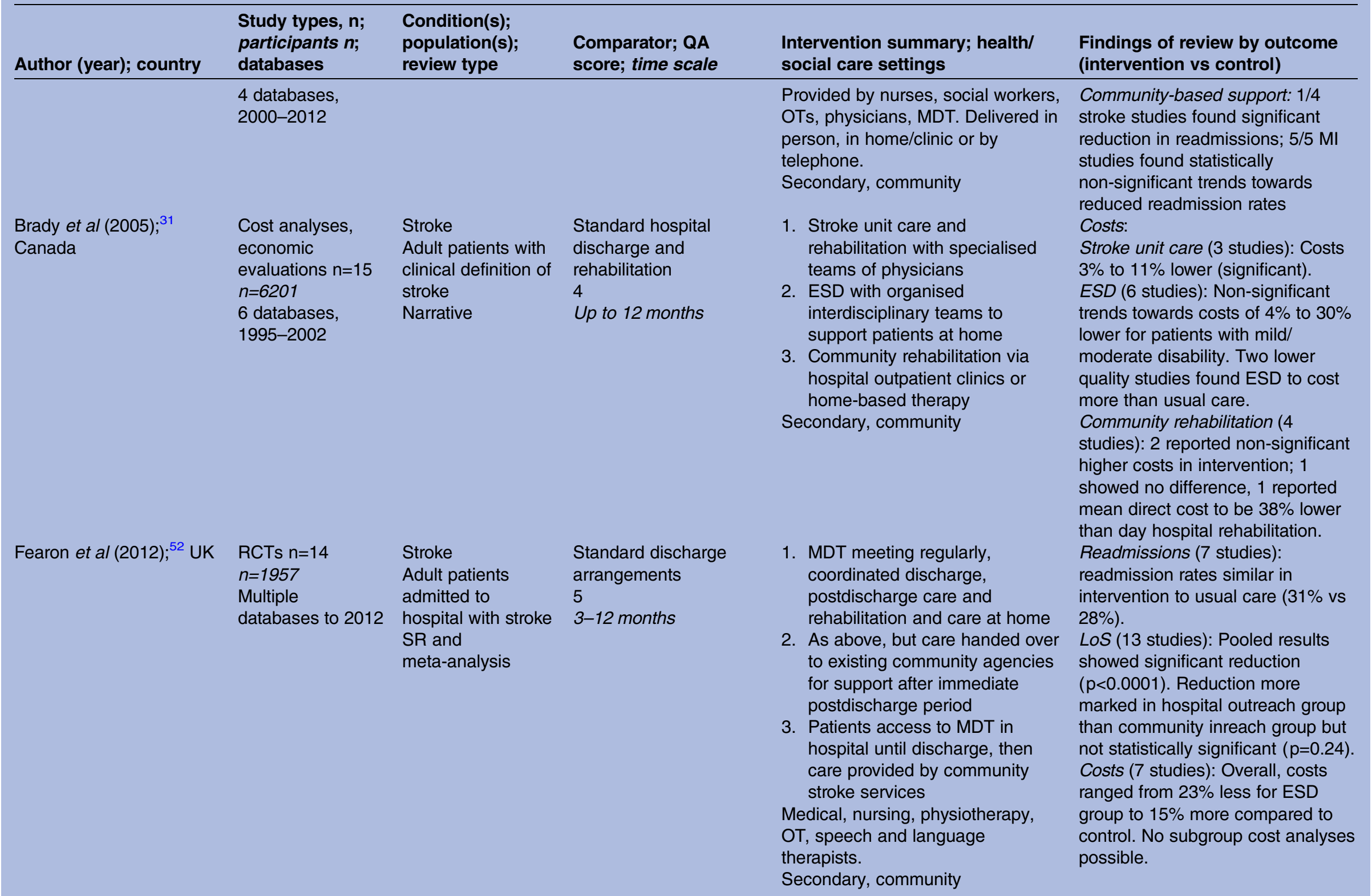

Continued 


\begin{tabular}{|c|c|c|c|c|c|}
\hline Author (year); country & $\begin{array}{l}\text { Study types, } \mathrm{n} ; \\
\text { participants } n \text {; } \\
\text { databases }\end{array}$ & $\begin{array}{l}\text { Condition(s); } \\
\text { population(s); } \\
\text { review type }\end{array}$ & $\begin{array}{l}\text { Comparator; QA } \\
\text { score; time scale }\end{array}$ & $\begin{array}{l}\text { Intervention summary; health/ } \\
\text { social care settings }\end{array}$ & $\begin{array}{l}\text { Findings of review by outcome } \\
\text { (intervention vs control) }\end{array}$ \\
\hline $\begin{array}{l}\text { Feltner et al (2014); }{ }^{53} \\
\text { USA }\end{array}$ & $\begin{array}{l}\text { RCTs } \mathrm{n}=47 \\
\text { Not specified } \\
5 \text { databases, } \\
2007-2013\end{array}$ & $\begin{array}{l}\text { Heart failure } \\
\text { Adult patients with } \\
\text { moderate to severe } \\
\text { HF } \\
\text { SR and } \\
\text { meta-analysis }\end{array}$ & $\begin{array}{l}\text { Standard discharge } \\
\text { arrangements } \\
4 \\
3-6 \text { months }\end{array}$ & $\begin{array}{l}\text { At least one of: } \\
\text { 1. Patient/caregiver education } \\
\text { 2. Multidisciplinary HF clinic visits } \\
\text { 3. Home visits by nurse or } \\
\text { pharmacist } \\
\text { 4. Telemonitoring } \\
\text { 5. Structured telephone support } \\
\text { 6. Transition coach/case } \\
\text { management } \\
\text { 7. Interventions for provider } \\
\text { continuity } \\
\text { Secondary, community }\end{array}$ & $\begin{array}{l}\text { Readmissions: } \\
\text { Home visits (2 studies): Significant } \\
\text { reduction in } 30 \text {-day all-cause } \\
\text { readmissions (RR 0.34, } 95 \% \mathrm{Cl} \\
0.19 \text { to } 0.62 \text { ) and } 3-6-\text { month } \\
\text { all-cause readmissions (RR } 0.75 \text {, } \\
95 \% \mathrm{Cl} 0.68 \text { to } 0.86 \text { ). } \\
\text { Significant reduction in } 3-6-\text {-month } \\
\text { HF-specific readmissions (1 study), } \\
\text { (RR 0.51, } 95 \% \mathrm{Cl} 0.31 \text { to } 0.82 \text { ). } \\
\text { Multidisciplinary HF clinics (2 } \\
\text { studies): Significant reduction in } \\
3 \text { to } 6 \text {-month all-cause readmission } \\
\text { (RR } 0.70,95 \% \mathrm{Cl} 0.55 \text { to } 0.89 \text { ). } \\
\text { No other intervention group had } \\
\text { any significant benefits. }\end{array}$ \\
\hline $\begin{array}{l}\text { Jeppesen et al (2012); }{ }^{54} \\
\text { Norway, UK, Australia }\end{array}$ & $\begin{array}{l}\text { RCTs } n=8 \\
n=870 \\
7 \text { databases, } \\
\text { inception-2010 } 1 \\
\text { inception-2012 }\end{array}$ & $\begin{array}{l}\text { COPD } \\
\text { Adult COPD } \\
\text { patients in ED with } \\
\text { acute exacerbation } \\
\text { SR and } \\
\text { meta-analysis }\end{array}$ & $\begin{array}{l}\text { Standard discharge } \\
\text { arrangements } \\
4.5 \\
6 \text { months }\end{array}$ & $\begin{array}{l}\text { Hospital at home: regular home } \\
\text { visits by a trained respiratory nurse } \\
\text { supported by the hospital team and } \\
\text { telephone support. } \\
\text { Secondary, community }\end{array}$ & $\begin{array}{l}\text { Readmissions ( } 8 \text { studies): } \\
\text { Significant reduction in intervention } \\
\text { group. } 9 \text { fewer readmissions per } \\
100 \text { compared to inpatient care } \\
\text { (RR } 0.76,95 \% \mathrm{Cl} 0.59 \text { to } 0.99 \text {, } \\
\mathrm{p}=0.04 \text { ). } \\
\text { Costs ( } 3 \text { studies): } 2 \text { reported } \\
\text { significant reduction in direct costs } \\
\text { for intervention; } 1 \text { reported } \\
\text { non-significant reduction. Authors } \\
\text { stress low quality of economic } \\
\text { evidence. }\end{array}$ \\
\hline $\begin{array}{l}\text { Lambrinou et al (2012); }{ }^{55} \\
\text { Greece }\end{array}$ & $\begin{array}{l}\text { RCTs } \mathrm{n}=19 \\
\text { Not specified } \\
3 \text { databases, } \\
\text { 2001-2009 }\end{array}$ & $\begin{array}{l}\text { Heart failure } \\
\text { Adult patients with } \\
\text { HF } \\
\text { SR and } \\
\text { meta-analysis }\end{array}$ & $\begin{array}{l}\text { Standard discharge } \\
\text { arrangements } \\
4 \\
\text { 3-35 months }\end{array}$ & $\begin{array}{l}\text { Nurse-driven predischarge phase, } \\
\text { incorporating discharge planning or } \\
\text { inpatient education and/or } \\
\text { evaluation. } \\
\text { Telephone follow-up; HF clinic } \\
\text { follow-up; home follow-up or a } \\
\text { combination. } \\
\text { Secondary, community }\end{array}$ & $\begin{array}{l}\text { Readmissions: } \\
\text { All-cause: Significantly reduced } \\
\text { across all interventions (RR } 0.85 \text {, } \\
95 \% \mathrm{Cl} 0.76 \text { to } 0.94) \text {. } \\
\text { Telephone, HF clinic, combined } \\
\text { settings all non-significant. } \\
\text { Home follow-up: RR } 0.80 \text { ( } 95 \% \mathrm{Cl} \\
0.70 \text { to } 0.91 \text { ). } \\
\text { HF-specific: Significantly reduced } \\
\text { across all interventions (RR } 0.68 \text {, } \\
95 \% \mathrm{Cl} 0.53 \text { to } 0.86 \text { ). }\end{array}$ \\
\hline
\end{tabular}




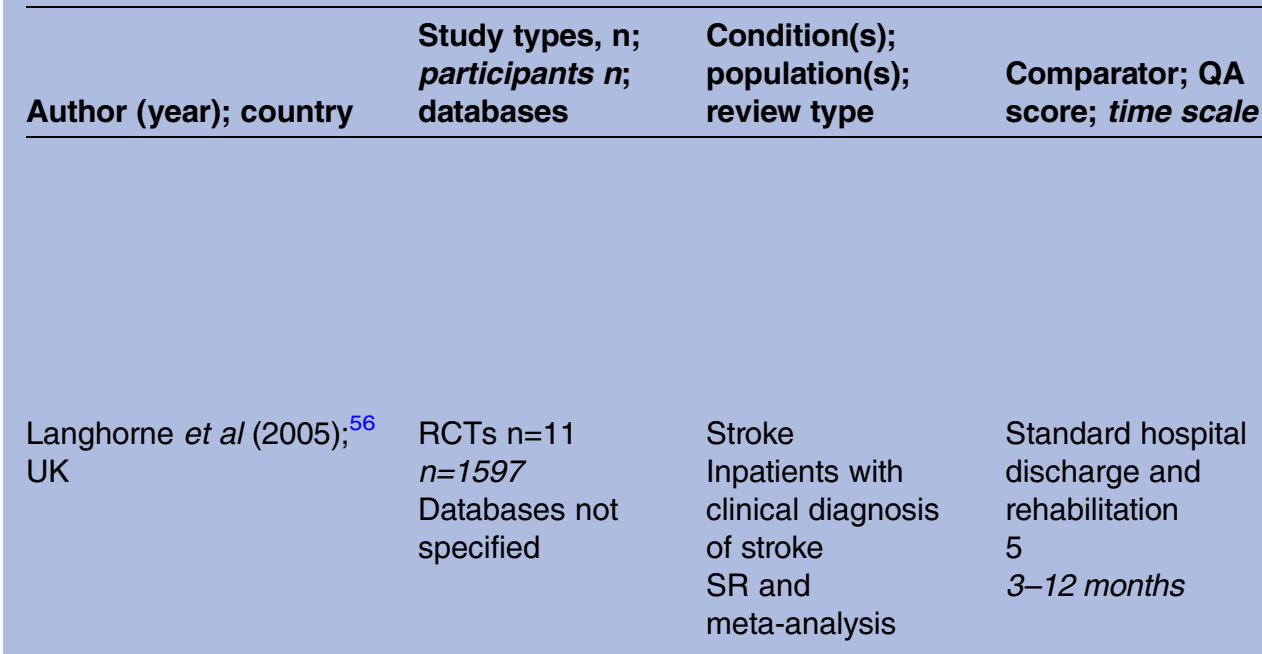

McMartin (2013); ${ }^{57}$

Canada
RCTs, SR, meta-analysis $\mathrm{n}=11$ Not specified 6 databases, 2004-2011
Chronic disease Adults with chronic diseases

SR and meta-analysis
Standard discharge arrangements Not specified
Intervention summary; health/ social care settings
Findings of review by outcome (intervention vs control)

Telephone follow-up (RR 0.65 , $95 \% \mathrm{Cl} 0.43$ to 1.00 )

HF clinic: Non-significant.

Home follow-up: RR $0.51(95 \% \mathrm{Cl}$ 0.33 to 0.79 )

Combined settings: RR 0.58 (95\%

$\mathrm{Cl} 0.45$ to 0.73 ).

Readmissions (5 studies): similar rates between intervention and control $(27 \%$ vs $25 \%$; OR 1.14 delivery; MDT coordinate discharge and postdischarge care and rehabilitation at home

2. ESD team coordination; postdischarge care by community agencies

3. No ESD team; MDT care in hospital, postdischarge care by uncoordinated community services/healthcare volunteers Medical staff, nurses, physiotherapy, therapists, assistant staff, social workers

Secondary, community

1. Discharge planning vs usual care

2. Comprehensive discharge planning with postdischarge support vs usual care, where postdischarge support could include home visits, telephone follow-up.

Secondary, community
$95 \% \mathrm{Cl} 0.80$ to 1.63 )

LOS (9 studies): Overall, significant reduction in intervention of 7.7 days (95\% Cl 4.2 to 10.7$)$.

Reduction greater for hospital outreach than community inreach ( 15 days, $95 \% \mathrm{Cl} 9$ to 22 vs 5 days, $95 \% \mathrm{Cl} 1$ to 9 ).

Controlling for stroke severity, greater reduction in severe vs moderate group ( 28 days, $95 \% \mathrm{Cl}$ 15 to 41 vs $4,95 \% \mathrm{Cl} 2$ to 6 ). Costs (5 studies): Intervention costs lower than control (range 4\% to $30 \%$ lower; median reduction $20 \%$ ). Significance not stated.

Readmissions:

Discharge planning (11 studies): Moderate evidence that intervention is effective (RR 0.85 , $95 \% \mathrm{Cl} 0.74$ to 0.97 ).

Discharge planning+postdischarge support. low quality evidence that this is more effective than

discharge planning alone.

LOS: Discharge planning more effective than usual care (mean reduction of 0.91 days, $95 \% \mathrm{Cl}$ 1.55 to 0.27 ). Discharge planning 


\begin{tabular}{lllll}
\hline & $\begin{array}{l}\text { Study types, } \mathrm{n} ; \\
\text { participants } n ; \\
\text { databases }\end{array}$ & $\begin{array}{l}\text { Condition(s); } \\
\text { population(s); } \\
\text { review type }\end{array}$ & $\begin{array}{l}\text { Comparator; QA } \\
\text { score; time scale }\end{array}$ & $\begin{array}{l}\text { Intervention summary; health/ } \\
\text { social care settings }\end{array}$ \\
\hline
\end{tabular}

$\begin{array}{llll}\text { Phillips et al (2005); }{ }^{59} & \text { RCTs } n=7 & \text { Heart failure } & \text { Not specified } \\ \text { USA } & n=949 & \text { Adult patients with } & 4 \\ & 5 \text { databases, } & \text { heart failure } & 3-12 \text { months } \\ & \text { inception-2004 } & \text { SR and } \\ & \text { meta-analysis }\end{array}$

Prieto-Centurion (2014); ${ }^{33} \quad$ RCTs $n=5$

USA

\section{4 databases} inception-2013

\section{COPD}

Exacerbation in previous 12 months

Narrative
Not specified

3 6 or 12 months social care settings

Findings of review by outcome (intervention vs control)

Costs (8 studies): 4 US based studies found significant costs reductions per patient per month of US $\$ 536$ (95\% CI -US\$956 to -US \$115). 4 non-US studies found no significant cost differences.

Readmissions:

Specialist nurse-led clinics to manage discharge transitions. Categorised by:

1. Complex interventions: discharge planning, postdischarge follow-up, no delay in continuity after discharge (3 studies)

2. Less complex: no discharge planning and/or fewer components (4 studies)

Secondary, community

Predischarge, postdischarge or bridging interventions across both periods.

Education, health counselling, action plans delivered via telephone, home visits or consultation with primary care providers

Primary, secondary, community Interventions grouped according to:
All-cause: 'Complex' programmes non-significant (RR $0.30,95 \% \mathrm{Cl}$ 0.04 to 2.60). 'Less complex' non-significant (RR $1.00,95 \% \mathrm{Cl}$ 0.86 to 1.17 )

HF-specific: 'Complex' programmes significant reduction (RR $0.09,95 \% \mathrm{Cl} 0.10$ to 0.65 .

'Less complex' significant reduction (RR 0.65, $955 \mathrm{Cl} 0.43$ to 1.00 ).

LoS: Complex interventions reduced LoS by 0.26 days compared to usual care (non-significant). Less complex interventions reduced LoS by 0.09 days (non-significant).

Costs: Only reported for complex interventions. 3 studies showed non-significant potential savings of US\$277 per patient per month. Readmissions:

All-cause: 2/5 studies showed significant reduction at 12 months: $45 \%$ vs $67 \%$ hospitalised

( $\mathrm{p}=0.028$ ).

COPD-specific: $1 / 5$ studies showed significant reduction at 12 months: $32 \%$ vs $50 \%$ hospitalised ( $p=0.01)$.

Costs: 


\begin{tabular}{|c|c|c|c|c|c|}
\hline Author (year); country & $\begin{array}{l}\text { Study types, } \mathrm{n} ; \\
\text { participants } n ; \\
\text { databases }\end{array}$ & $\begin{array}{l}\text { Condition(s); } \\
\text { population(s); } \\
\text { review type }\end{array}$ & $\begin{array}{l}\text { Comparator; QA } \\
\text { score; time scale }\end{array}$ & $\begin{array}{l}\text { Intervention summary; health/ } \\
\text { social care settings }\end{array}$ & $\begin{array}{l}\text { Findings of review by outcome } \\
\text { (intervention vs control) }\end{array}$ \\
\hline $\begin{array}{l}\text { Tummers et al (2012); }{ }^{34} \\
\text { Netherlands }\end{array}$ & $\begin{array}{l}\text { RCTs, CCTs, } \\
n=15 \\
n=3536 \\
2 \text { databases, } \\
\text { inception-2011 }\end{array}$ & $\begin{array}{l}\text { Stroke } \\
\text { Adult patients who } \\
\text { had stroke } \\
\text { Narrative }\end{array}$ & $\begin{array}{l}\text { Standard hospital } \\
\text { discharge and } \\
\text { rehabilitation } \\
3 \\
\text { 3-12 months }\end{array}$ & $\begin{array}{l}\text { 1. ESD by MDT, home-based } \\
\text { rehabilitation } \\
\text { 2. Stroke unit care with MDTs to } \\
\text { reach rehabilitation goals before } \\
\text { discharge } \\
\text { 3. Stroke service via network of } \\
\text { providers organising services in } \\
\text { all follow-up stages } \\
\text { Primary, secondary, community }\end{array}$ & $\begin{array}{l}\text { Group } 1 \text { ( } 4 \text { studies): } 3 \text { reported } \\
\text { non-significant increases in } \\
\text { intervention; } 1 \text { reported no } \\
\text { difference between groups. } \\
\text { Group } 2 \text { ( } 2 \text { studies): Both found } \\
\text { stroke units to be more expensive } \\
\text { than conventional care (borderline } \\
\text { significance). } \\
\text { Group } 3 \text { ( } 3 \text { studies): } 2 \text { reported a } \\
\text { cost reduction in intervention } \\
\text { group. }\end{array}$ \\
\hline $\begin{array}{l}\text { Winkel et al (2008); }{ }^{35} \\
\text { Denmark, Sweden }\end{array}$ & $\begin{array}{l}\text { RCTs } \mathrm{n}=17 \\
n=1122 \\
5 \text { databases, } \\
\text { inception-2005 }\end{array}$ & $\begin{array}{l}\text { Stroke } \\
\text { Adult patients who } \\
\text { had been living at } \\
\text { home before a } \\
\text { stroke } \\
\text { Narrative }\end{array}$ & $\begin{array}{l}\text { Standard discharge } \\
\text { arrangements } \\
4 \\
1-12 \text { months }\end{array}$ & $\begin{array}{l}\text { Delivered by MDTs which all } \\
\text { included physiotherapists and OTs. } \\
\text { Some also included nurse, social } \\
\text { worker, GP and other specialist } \\
\text { expertise, eg, geriatrician. } \\
\text { 1. ESD with hospital teams } \\
\text { providing home rehabilitation } \\
\text { after discharge } \\
\text { 2. ESD with no direct rehabilitation } \\
\text { from hospital teams } \\
\text { 3. Community-based rehabilitation } \\
\text { after discharge } \\
\text { Primary, secondary, community }\end{array}$ & $\begin{array}{l}\text { Readmissions: } \\
\text { Group } 1 \text { ( } 3 \text { studies): No difference } \\
\text { between groups. } \\
\text { Group } 2 \text { ( } 2 \text { studies): No difference } \\
\text { between groups. } \\
\text { Group } 3 \text { ( } 1 \text { study): No difference } \\
\text { between groups. } \\
\text { Costs: } \\
\text { Group } 1 \text { ( } 2 \text { studies): Intervention } \\
\text { costs significantly lower than } \\
\text { control at } 3 \text { and } 12 \text { months. } \\
\text { Group } 2 \text { ( } 1 \text { study): 'Some' evidence } \\
\text { that intervention costs are lower } \\
\text { than control in } 12 \text { months after } \\
\text { stroke. } \\
\text { Group } 3 \text { ( } 1 \text { study): Costs for the } \\
\text { most independent patients were } \\
\text { lowest when rehabilitated in } \\
\text { hospital rather than home. } \\
\text { Interventions most cost-effective } \\
\text { when delivered by hospital MDT. }\end{array}$ \\
\hline $\begin{array}{l}\text { Yu et al (2006); }{ }^{36} \text { Hong } \\
\text { Kong }\end{array}$ & $\begin{array}{l}\text { RCTs } n=21 \\
n=4445 \\
3 \text { databases, } \\
1995-2005\end{array}$ & $\begin{array}{l}\text { Heart Failure } \\
\text { Adult patients with } \\
\text { heart failure } \\
\text { Narrative }\end{array}$ & $\begin{array}{l}\text { Not specified } \\
4 \\
3-50 \text { months }\end{array}$ & $\begin{array}{l}\text { Postdischarge interventions } \\
\text { delivered via home visits, HF clinic } \\
\text { visits and/or telephone. } \\
\text { Interventions comprised } \\
\text { multidisciplinary care, case } \\
\text { management and structured } \\
\text { discharge planning and all included }\end{array}$ & $\begin{array}{l}\text { Readmissions: } 11 \text { 'effective' } \\
\text { programmes had significant } \\
\text { reductions ranging from } 29 \% \text { to } \\
85 \% .10 \text { others demonstrated no } \\
\text { significant changes. Effective } \\
\text { programmes included an } \\
\text { in-hospital phase, patient }\end{array}$ \\
\hline
\end{tabular}




\begin{tabular}{llll}
\hline Author (year); country & $\begin{array}{l}\text { Study types, } \mathbf{n} ; \\
\text { participants } n ; \\
\text { databases }\end{array}$ & $\begin{array}{l}\text { Condition(s); } \\
\text { population(s); } \\
\text { review type }\end{array}$ & $\begin{array}{l}\text { Comparator; } \\
\text { score; time } \text { s }\end{array}$ \\
& & & \\
& & & \\
& & & \\
& & & \\
& & & \\
Complex interventions & & COPD & Not specified \\
Dickens et al (2014);60 & RCTs n=32 & Adult patients with & 4 \\
UK & $n=3941$ & COPD & $1-24$ months \\
& 5 databases, \\
inception-2013 & SR and \\
meta-analysis &
\end{tabular}

Martinez-González et al (2014); ${ }^{70}$ Switzerland

$\mathrm{SR}$

meta-analyses

$\mathrm{n}=27$

Not specified

4 databases,

inception-2012
Chronic disease Not specified Adult patients with 3

chronic diseases Not specified Review of reviews
Included any interventions based on disease management, case management, managed care, comprehensive care, multidisciplinary care, coordinated care, team care, CCMs. Primary, secondary, community
Findings of review by outcome (intervention vs control)

education, self-care, surveillance and deterioration management. Involvement of cardiac nurses and cardiologists associated with increased likelihood of successful intervention.

Costs: 8 'effective' programmes did cost analysis, 7 of which showed a cost saving for the intervention over usual care.

A\&E use: Pooled effects showed interventions associated with $32 \%$ reduction (OR $0.68,95 \% \mathrm{Cl} 0.57$ to $0.80)$. Subgroups:

General education (28 studies): OR $0.66,95 \% \mathrm{Cl} 0.55$ to 0.81 .

Exercise (11 studies): OR 0.60

therapy, social or organisational interventions. Delivered at home, in community, hospital or doctor clinic or combination of these.

Primary, secondary, community

$95 \% \mathrm{Cl} 0.48$ to 0.76 .

Relaxation (4 studies): OR 0.48 ,

$95 \% \mathrm{Cl} 0.33$ to 0.70 .

Non-significant trends for interventions including skills training ( $\mathrm{p}=0.35,13$ studies), relapse prevention $(p=0.12,11$ studies).

Admissions: 10/17 reviews demonstrated reduced admissions Readmissions: $7 / 12$ reviews demonstrated reduced readmissions

LOS: 9/13 reviews demonstrated shorter length of stay A\&E use: 6/11 reviews showed reduced rates of $E D$ visits

Costs: $3 / 17$ reviews demonstrated cost reductions

Continued 
Multidisciplinary teams Health Quality Ontario (2012); ${ }^{71}$ Canada

Health Quality Ontario (2013); ${ }^{37}$ Canada
SR and

meta-analyses $\mathrm{n}=24$

Not specified

6 databases, 2008-2011

SR, RCTs, observational studies $\mathrm{n}=20$ Not specified 5 databases, 2002-2011
Condition(s);

population(s);

review type

Comparator; QA

score; time scale

Heart failure Adults with at least one HF secondary

Not specified care admission

SR and

meta-analysis

5

6-24 months
Heart failure,

COPD

Adult patients with

heart failure or

COPD

Review of reviews
Usual care in general practice

Not specified
Intervention summary; health/ social care settings

All led by professionals from secondary or tertiary care.

Interventions grouped as:

1. Case management, telephone and home visits

2. Specialist nurse-led HF clinics

3. Multidisciplinary interventions to bridge the gap between acute and home settings

Secondary, community
Chronic disease Not specified Adult patients with 3 one or more chronic diseases

Narrative
3 Not specified
Interventions to provide formalised links between primary and specialist care via disease-specific education, medication review, physical activity and lifestyle counselling, self-care and follow-up. Delivered by intermediate care teams including GPs, specialists, nurses, social workers, pharmacists, dieticians. Primary, secondary, community Informational, management and relational continuity. Assessed by:

1. Duration (length of relationship)

2. Density (number of visits with same provider in a set period)

3. Dispersion (visits with distinct providers)

4. Sequence (order of seeing providers).

Primary, community
Findings of review by outcome (intervention vs control)

Readmissions:

HF-specific (12 studies): Overall, significantly reduced (OR 0.57 , $95 \% \mathrm{Cl} 0.43$ to $0.75, p<0.0001$ ). Subgroups:

Group 1: Significant reduction at

6 months (3 studies) and 12 months ( 7 studies). OR 0.64 $(95 \% \mathrm{Cl} 0.46$ to 0.88$)$ and $\mathrm{OR} 0.47$ ( $95 \% \mathrm{Cl} 0.30$ to 0.76 ), respectively. Group 2: No difference between groups.

Group 3 (2 studies): Significant reduction OR $0.45,95 \% \mathrm{Cl} 0.28$ to 0.72 ). All-cause also significantly reduced with multidisciplinary interventions: (OR $0.46,95 \% \mathrm{Cl}$ 0.30 to 0.69 ).

Admissions:

All-cause ( 7 studies)

Non-significant 4\% RR reduction after 1 year (low quality).

COPD-specific (4 studies).

Significant 25\% RR reduction after 1 year (moderate quality).

HF-specific (6 studies).

Non-significant $14 \%$ RR reduction after 1 year (low quality).

Admissions:

Three studies. None reported any significant differences between intervention and control groups (low quality). 


\begin{tabular}{|c|c|c|c|c|c|}
\hline Author (year); country & $\begin{array}{l}\text { Study types, } n \text {; } \\
\text { participants } n ; \\
\text { databases }\end{array}$ & $\begin{array}{l}\text { Condition(s); } \\
\text { population(s); } \\
\text { review type }\end{array}$ & $\begin{array}{l}\text { Comparator; QA } \\
\text { score; time scale }\end{array}$ & $\begin{array}{l}\text { Intervention summary; health/ } \\
\text { social care settings }\end{array}$ & $\begin{array}{l}\text { Findings of review by outcome } \\
\text { (intervention vs control) }\end{array}$ \\
\hline Holland et al (2005); ${ }^{62}$ UK & $\begin{array}{l}\text { RCTs } n=30 \\
n=815813 \\
\text { databases } \\
\text { inception-2004 }\end{array}$ & $\begin{array}{l}\text { Heart failure } \\
\text { Adult patients with } \\
\text { congestive heart } \\
\text { failure } \\
\text { SR and } \\
\text { meta-analysis }\end{array}$ & $\begin{array}{l}\text { Not specified } \\
5 \\
\text { Not specified }\end{array}$ & $\begin{array}{l}\text { Interventions with management by } \\
\text { an MDT that included medical input } \\
\text { plus one or more of specialist } \\
\text { nurse, pharmacist, health educator, } \\
\text { dietician or social worker: } \\
\text { 1. Education/self-management } \\
\text { home visits } \\
\text { 2. Telephone follow-up only } \\
\text { 3. Intervention during hospital } \\
\text { admission or hospital clinic } \\
\text { attendance } \\
\text { Primary, secondary, community }\end{array}$ & $\begin{array}{l}\text { Admissions: } \\
\text { All-cause ( } 21 \text { studies): Significant } \\
\text { reduction in intervention (RR } 0.87 \text {, } \\
95 \% \mathrm{Cl} 0.79 \text { to } 0.95, \mathrm{p}=0.002) \text {. } \\
\text { Significant heterogeneity. } \\
\text { HF-specific ( } 16 \text { studies): Significant } \\
\text { reduction in intervention (RR } 0.70 \text {, } \\
95 \% \mathrm{Cl} 0.61 \text { to } 0.81, \mathrm{p}<0.0001) \text {. } \\
\text { LoS ( } 10 \text { studies): Significant } \\
\text { reduction in mean inpatient days of } \\
1.9 \text { in intervention ( } 95 \% \mathrm{Cl} 0.71 \text { to } \\
3.1 \text { ). } \\
\text { Home-based interventions reduced } \\
\text { mean days in hospital. } \\
\text { Interventions solely delivered in } \\
\text { hospital, clinic or primary care } \\
\text { showed no significant benefits. }\end{array}$ \\
\hline $\begin{array}{l}\text { Koshman et al (2008); } \\
\text { Canada }\end{array}$ & $\begin{array}{l}\text { RCTs } n=12 \\
n=2060 \\
10 \text { databases } \\
\text { inception-2007 }\end{array}$ & $\begin{array}{l}\text { Heart failure } \\
\text { Adult patients with } \\
\text { heart failure } \\
\text { SR and } \\
\text { meta-analysis }\end{array}$ & $\begin{array}{l}\text { Heart failure care } \\
\text { without pharmacist } \\
\text { involvement } \\
4 \\
6-12 \text { months }\end{array}$ & $\begin{array}{l}\text { Pharmacists providing HF and } \\
\text { medication education through } \\
\text { self-monitoring support, compliance } \\
\text { facilitation. } \\
\text { Either via directed care where } \\
\text { pharmacist is the key driver, or } \\
\text { collaborative care with pharmacist } \\
\text { as part of MDT. } \\
\text { Secondary, community }\end{array}$ & $\begin{array}{l}\text { Admissions: } \\
\text { All-cause ( } 11 \text { studies): Significant } \\
\text { reduction (OR } 0.71,95 \% \mathrm{Cl} 0.54 \text { to } \\
0.94 \text { ). No difference between } \\
\text { directed and collaborative care } \\
\text { model. } \\
\text { HF-specific ( } 11 \text { studies): Significant } \\
\text { reduction (OR } 0.69,95 \% \mathrm{Cl} 0.51 \text { to } \\
0.94 \text { ). Collaborative care model } \\
\text { associated with greater reduction in } \\
\text { HF-specific admission than } \\
\text { directed care (OR } 0.42,95 \% \mathrm{Cl} \\
0.24 \text { to } 0.74 \text { vs OR } 0.89,95 \% \mathrm{Cl} \\
0.68 \text { to } 1.17, \mathrm{p}=0.02 \text { ). }\end{array}$ \\
\hline McAlister (2004); ${ }^{64}$ UK & $\begin{array}{l}\text { RCTs } n=29 \\
n=5039 \\
7 \text { databases, } \\
\text { inception-2003 }\end{array}$ & $\begin{array}{l}\text { Heart failure } \\
\text { Adult patients with } \\
\text { HFSR and } \\
\text { meta-analysis }\end{array}$ & $\begin{array}{l}\text { Not specified } \\
4 \\
1-12 \text { months }\end{array}$ & $\begin{array}{l}\text { 1. Multidisciplinary HF clinic } \\
\text { 2. MDT providing specialised } \\
\text { follow-up outside hospital } \\
\text { 3. Telephone follow-up with } \\
\text { primary care attendance in the } \\
\text { event of deterioration } \\
\text { 4. Self-care education } \\
\text { 5. Primary, community }\end{array}$ & $\begin{array}{l}\text { Admissions: } \\
\text { Groups } 1+2 \text { : HF hospitalisation } \\
\text { significantly reduced (RR } 0.74 \text {, } \\
95 \% \mathrm{Cl} 0.63 \text { to } 0.87 \text { ); all-cause } \\
\text { hospitalisation significantly reduced } \\
\text { (RR } 0.81,95 \% \mathrm{Cl} 0.71 \text { to } 0.92 \text { ). } \\
\text { Group } 3 \text { : HF hospitalisation } \\
\text { significantly reduced (RR } 0.66 \text {, }\end{array}$ \\
\hline
\end{tabular}

5. Primary, community 


\begin{tabular}{lllll}
\hline & $\begin{array}{l}\text { Study types, } \mathrm{n} ; \\
\text { participants } n ; \\
\text { databases }\end{array}$ & $\begin{array}{l}\text { Condition(s); } \\
\text { population(s); } \\
\text { review type }\end{array}$ & $\begin{array}{l}\text { Comparator; QA } \\
\text { score; time scale }\end{array}$ & $\begin{array}{l}\text { Intervention summary; health/ } \\
\text { social care settings }\end{array}$ \\
\hline
\end{tabular}
(intervention vs control)

$95 \% \mathrm{Cl} 0.52$ to 0.83 ). All-cause hospitalisation no significant effect. Group 4: HF hospitalisation significantly reduced (RR 0.66 , $95 \% \mathrm{Cl} 0.52$ to 0.83 ). All-cause hospitalisation significantly reduced (RR $0.73,95 \% \mathrm{Cl} 0.57$ to 0.93 ). Costs (18 studies): 15 found cost

Medical Advisory

Secretariat (2009); ${ }^{65}$

Canada

\section{RCTs $\mathrm{n}=8$}

$n=2692$

4 databases inception-2008
Roccaforte et al (2005); $66 \quad$ RCTs $n=33$ Canada
Not specified

4 databases, 1980-2004
Heart failure Adult patients with HF

SR and meta-analysis

Heart failure HF patients followed up in outpatient setting SR and meta-analysis
Care not provided by multiple practitioners At least 12 months eferral to family physician or home care services after discharge 5 3-22 months
All included a team of nurse and physician and/or general practitioner, one of which specialised in HF management. Varying combinations of disease-specific education, diet, lifestyle, exercise counselling, self-care support, follow-up. Delivered directly (clinic based programme) or indirectly (telephone based, physician supervised, nurse-led).

Primary, secondary, community savings; 3 found neutral costs.

Readmissions:

All-cause (7 studies):

Non-significant increase in intervention group. Significant 12\% reduction when care delivered through a direct (clinic) model. HF-specific (6 studies):

Non-significant RR reduction of $14 \%$ in intervention.

LoS (7 studies): Patients receiving intervention generally had shorter LoS whether measured as mean duration (4 studies) or total bed days ( 3 studies)

A\&E use ( 1 study): $77 \%$ of intervention patients vs $84 \%$ of control patients had an ED visit within 12 months ( $p=0.029$ ). Readmissions:

1. Multidisciplinary approach, starting during hospitalisation, continuing for up to 12 months postdischarge, delivered by various professionals

2. Approach centred on specific health professionals, eg, HF

specialist nurses or case managers, focused on particular care components, eg, therapy adherence

All-cause: $7 / 32$ studies found significant reductions (OR 0.76 $95 \% \mathrm{Cl} 0.69$ to 0.94 ).

HR-specific: $8 / 20$ found significant reductions (OR 0.58, 95\% $\mathrm{Cl} 0.50$ to 0.67$)$. By subgroup:

Group 1: All-cause and HF-specific readmissions significantly reduced

(OR $0.58,95 \% \mathrm{Cl} 0.47$ to 0.71 )

and (OR $0.58,95 \% \mathrm{Cl} 0.45$ to

Primary, secondary, community 0.75$)$, respectively. 


\begin{tabular}{lllll}
\hline & $\begin{array}{l}\text { Study types, } \mathrm{n} ; \\
\text { participants } \mathrm{n} ; \\
\text { databases }\end{array}$ & $\begin{array}{l}\text { Condition(s); } \\
\text { population(s); } \\
\text { review type }\end{array}$ & $\begin{array}{l}\text { Comparator; QA } \\
\text { score; time scale }\end{array}$ & $\begin{array}{l}\text { Intervention summary; health/ } \\
\text { social care settings }\end{array}$ \\
\hline
\end{tabular}

Findings of review by outcome (intervention vs control)

Group 2: All-cause and HF-specific readmissions significantly reduced (OR $0.82,95 \% \mathrm{Cl} 0.74$ to 0.91 ) and ()R $0.61,95 \% \mathrm{Cl} 0.51$ to 0.73 ), respectively.

LoS (12 studies): Significant

reduction of -1.49 days $(95 \% \mathrm{Cl}$ -2.03 to -0.95 days).

Sikich (2012); ${ }^{38}$ Canada

$\mathrm{HTAs}$,
$\mathrm{n}=6$

$n=1370$

6 databases,

1995-2010
COPD

Adult patients with

COPD

Narrative

Care not provided by multiple practitioners 4
Smith et al (2007); Ireland
RCTs, CCTs, before/after, time series $\mathrm{n}=20$ Not specified

7 databases, inception-2006
Chronic disease

Patients in a primary and secondary shared care service Narrative
Care not provided by multiple practitioners 5 Not specified
Interventions based on CCM components, delivered by various professionals as a team in one organisation or range of

organisations together as a unique team.

Most teams included a respiratory specialist and/or a physician Primary, secondary, community
Liaison meetings attended by specialists and primary care staff to discuss and plan ongoing patient management; shared care record carried by the patient,

computer-assisted shared care and email with data available to primary and secondary care Primary, secondary 
Self-management

Franek (2013); $;{ }^{41}$ Canada SR, RCTs,

meta-analyses

$\mathrm{n}=10$

$n=6074$

5 databases, 2000-2012

Harrison et al (2015); ${ }^{67}$

Canada

\section{RCTs $\mathrm{n}=7$}

$n=1115$

7 databases,

inception-2014

RCTs $\mathrm{n}=6$

$n=857$

Canada
Chronic disease

Care from the usual

Adult patients with provide

Narrative

4-12 months

COPD

Adult patients

hospitalised

following acute

exacerbation

SR and

meta-analysis

Heart failure

Adult patients with

Not specified

3.5

2 weeks-12 months chronic disease $\quad 3.5$

Comparator; QA

Not specified

-24 months
Not specified

4

3-12 months
Intervention summary; health/ social care settings

Any intervention to improve

outcomes for patients with

multimorbidity in primary or

community care delivered by an

MDT.

6 studies assessed MDT

interventions.

Primary, community
Stanford chronic disease programme: 6 weekly 2.5 hou sessions with 10-15 participants, in community settings, with volunteer lay facilitators assisting patients to make their own management choices and reach self-selected goals.

Primary, secondary, community

Action plans involving symptom monitoring, education and at least 2 of 7 self-management skills (self-efficacy, problem solving, resource use, collaboration,

emotional/role management, goal setting)

Delivered by nurses when patient

is in hospital, or within 1 month of discharge.

Secondary, community

Education and limited follow-up: patients taught to monitor condition and recognise symptom
Findings of review by outcome (intervention vs control)

Admissions (5 studies): One study

found significant reduction in admissions with intervention; 4 found no difference between

groups.

Costs (4 studies): One reported no difference between groups; one

had no results available; one

reported a non-significant marginal benefit for intervention, one reported net savings in intervention costs but did not account for other costs.

Admissions (3 studies): No significant difference in admission rates between intervention and control in any study (low quality evidence).

LoS (5 studies): None showed any significant differences between

groups at 6 months.

A\&E use (5 studies): No significant differences between groups.

Readmissions (5 studies).

Meta-analysis found no significant differences at 12 months between intervention and control groups in terms of the number of patients readmitted to hospital. Mean difference $1.32, \mathrm{Cl} 0.71$ to 2.46 $(p=0.38)$.

Readmissions:$$
\text { (1) }
$$ 


\begin{tabular}{|c|c|c|c|c|}
\hline Author (year); country & $\begin{array}{l}\text { Study types, } \mathrm{n} ; \\
\text { participants } n \text {; } \\
\text { databases }\end{array}$ & $\begin{array}{l}\text { Condition(s); } \\
\text { population(s); } \\
\text { review type }\end{array}$ & $\begin{array}{l}\text { Comparator; QA } \\
\text { score; time scale }\end{array}$ & $\begin{array}{l}\text { Intervention summary; health/ } \\
\text { social care settings }\end{array}$ \\
\hline & $\begin{array}{l}6 \text { databases, } \\
\text { inception-2005 }\end{array}$ & $\begin{array}{l}\text { HF } \\
\text { Narrative }\end{array}$ & & $\begin{array}{l}\text { exacerbation; follow-up phone call } \\
\text { and face to face or digital } \\
\text { education. } \\
\text { Delivered by nurses or AHPs. } \\
\text { Secondary, community }\end{array}$ \\
\hline
\end{tabular}

Findings of review by outcome (intervention vs control)

All-cause (5 studies): Significant reduction in intervention (OR 0.59 , $95 \% \mathrm{Cl} 0.44$ to 0.80 ).

HF-specific (3 studies): Significant reduction in intervention (OR 0.44 , $95 \% \mathrm{Cl} 0.27$ to 0.71 )

Costs ( 3 studies): All reported annual savings for intervention vs usual care of between US $\$ 1300$ and US\$7515.

Smith et al (2012); Ireland

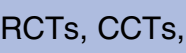

before/after, time

series

$\mathrm{n}=10$

$n=3357$

9 databases,

various-2011

RCTs, controlled

trials, $n=31$

$n=3688$

6 databases

1995-2011

Zwerink et al (2014); ${ }^{68}$

Netherlands

\section{Chronic disease Not specified}

multimorbidity in

primary care or

community

Narrative

Not specified
COPD

Patients with clinical diagnosis of COPD

$\mathrm{SR}$ and

meta-analysis
5 2-24 months

Any patient-orientated intervention to promote self-management in patients with multimorbidity in primary or community care.

Four studies assessed self-management interventions. Primary, community

2-24 months

Structured interventions to improve self-health and self-management skills.

At least 2 of action plan, exercise programme, smoking cessation, dietary advice, medication review, coping with breathlessness advice, CBT, motivational interviewing, goal setting, feedback.

Primary, secondary, community

Admissions (2 studies): One reported significant reduction in favour of intervention. The other found no difference between groups.

Costs (2 studies): One reported cost savings per participant due to reduction in admission rates in intervention group. The other found no difference between groups.

Admissions:

All-cause (6 studies): 310 patients per 1000 admitted within

12 months in intervention vs 428 control. Statistically significant reduction (OR $0.60,95 \% \mathrm{Cl} 0.40$ to 0.89 ).

COPD-specific (9 studies): 190 patients per 1000 admitted within 12 months in intervention vs 293 control. Statistically significant reduction (OR $0.57,95 \% \mathrm{Cl} 0.43$ to $0.75)$.

LoS (5 studies): No differences between groups.

${ }^{*}$ Smith et $a 4^{40}$ listed twice due to focus on MDT interventions and self-management interventions.

AHP, allied health professional; CBT, cognitive-behavioural therapy; CCM, chronic care model; CCT, controlled clinical trial; ED, emergency department; ESD, early supported discharge; HF,

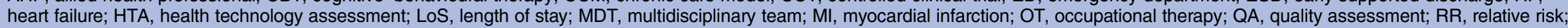
SMD, standardised mean difference; SR, systematic review. 
Table 3 Intervention groupings

\begin{tabular}{|c|c|}
\hline Category & Description of intervention \\
\hline $\begin{array}{l}\text { Case management } \\
(\mathrm{n}=8)^{23-27} 43-45\end{array}$ & $\begin{array}{l}\text { Based on implementation of a collaborative process between one or more care } \\
\text { coordinators or case managers and the patient, to assess, plan and facilitate service } \\
\text { delivery for patients with chronic diseases, particularly when transitions across healthcare } \\
\text { settings are required }\end{array}$ \\
\hline $\begin{array}{l}\text { Chronic care model } \\
(\mathrm{n}=9)^{28} 2946-5169\end{array}$ & $\begin{array}{l}\text { Model that identifies six modifiable elements of healthcare systems: (1) organisational } \\
\text { support, addressing organisational culture and leadership, (2) clinical information systems } \\
\text { to organise patient, population and provider data, (3) delivery system design to address } \\
\text { composition and function of the care team and follow-up management, (4) decision } \\
\text { support to increase provider access to evidence-based guidelines and specialists for } \\
\text { collaboration, (5) self-management support to provide tailored education, skills training, } \\
\text { psychosocial support and goal-setting and (6) community resources to provide peer } \\
\text { support, care coordination and community-based interventions }\end{array}$ \\
\hline $\begin{array}{l}\text { Discharge management } \\
(n=15)^{30-36} 52-59\end{array}$ & $\begin{array}{l}\text { Interventions designed to facilitate effective transitions from hospital care to other settings } \\
\text { Typically includes a predischarge phase of support, transitional care for the move } \\
\text { between the hospital and community/home setting and postdischarge follow-up and } \\
\text { monitoring, often incorporating rehabilitation or reablement support }\end{array}$ \\
\hline $\begin{array}{l}\text { Complex interventions } \\
(n=3)^{60} 6170\end{array}$ & $\begin{array}{l}\text { Two reviews assessed a range of interventions rather than focusing on a single } \\
\text { intervention or service model }\end{array}$ \\
\hline $\begin{array}{l}\text { Multidisciplinary teams } \\
(\mathrm{n}=10)^{37-40} 62-6671\end{array}$ & $\begin{array}{l}\text { Interventions comprising teams composed of multiple health and/or social care } \\
\text { professionals working together to provide care for people with complex needs. Teams } \\
\text { typically included condition-specific expertise, nurses, occupational therapists, } \\
\text { physiotherapists, social workers, GPs and occasionally pharmacists or case managers }\end{array}$ \\
\hline $\begin{array}{l}\text { Self-management } \\
(\mathrm{n}=5)^{40-42} 6768\end{array}$ & $\begin{array}{l}\text { Interventions designed to provide patient support, typically via tailored education to inform } \\
\text { the patient about their condition(s), recognising signs and symptoms of disease } \\
\text { exacerbation, dietary and lifestyle advice and/or condition-specific education supporting } \\
\text { medication adherence }\end{array}$ \\
\hline
\end{tabular}

association between the intervention and outcomes assessed. Of nine reviews focusing on interventions comprising one or more components of the CCM, six reported positive findings for at least one outcome. $^{28} 46-485069$ All CCM reviews reported that interventions with multiple components were significantly more effective than single component interventions at reducing admission rates, ${ }^{46} \quad 49 \quad 50 \quad 69$ with reductions of 22-32\% observed in reviews that performed meta-analysis. Multicomponent interventions were also successful in reducing readmissions by $15-30 \%,{ }^{47}$ length of hospital stay by $2-4$ days ${ }^{46} 48$ and A\&E visits by $42 \%{ }^{46}$

Fifteen reviews assessed discharge management interventions, predominantly focusing on readmission rates and length of stay (LoS). Six reviews reported significant reductions in readmission rates for patients with heart failure, ${ }^{53} 555859 \mathrm{COPD}^{54}$ and general chronic diseases. ${ }^{57}$ Reductions ranged from $15 \%{ }^{55}$ to $66 \%{ }^{53}$ In contrast, discharge management for patients who had stroke was notably ineffective in reducing readmission rates, ${ }^{32} 355256$ although LoS reduced by 7.7 days in one stroke review. ${ }^{56}$ Three reviews assessed complex interventions. One demonstrated a $32 \%$ reduction in A\&E use, ${ }^{60}$ another reported a $43 \%$ reduction in heart failure-related readmissions ${ }^{61}$ and a review of reviews reported positive findings for admissions, readmissions, LoS and A\&E use (no effect sizes given). ${ }^{70}$
Ten reviews assessed MDT interventions. Although team composition varied, MDT were generally effective when used for patients with single conditions, showing a $26-31 \%$ reduction in admission rates for heart failure ${ }^{62-64}$ and a 33\% RRR for admissions in patients with COPD. ${ }^{38}$ MDT were also associated with a $42 \%$ reduction in heart failure readmissions, ${ }^{66}$ a 2 -day reduction in LoS, ${ }^{626566}$ significantly reduced A\&E use ${ }^{65}$ and significantly lower healthcare costs. ${ }^{64}$ Conversely, MDT for general chronic disease management showed mixed effectiveness or no significant association for any outcomes, ${ }^{3739} 40$ suggesting that the crucial component of an effective MDT is the inclusion of condition-specific specialist expertise in the team skill mix. Finally, five reviews assessed self-management interventions. Three showed either mixed findings ${ }^{40}$ or no association between intervention and outcomes assessed. ${ }^{41} 67$ The remaining two demonstrated significant reductions in readmission rates and healthcare costs for patients with heart failure ${ }^{42}$ and significantly lower admission rates for COPD. ${ }^{68}$

\section{Hospital admissions}

Emergency admission rates were assessed in 21 reviews across five intervention categories (table 4). Eleven reviews reported significantly reduced admissions, $^{38} 45464850$ 62-64 68-70 with all but two positive reviews focusing on heart failure ${ }^{45} 62-64$ or 
Table 4 Summary of effectiveness for each outcome by review and intervention category

\begin{tabular}{|c|c|c|c|c|c|}
\hline Review & Admissions & Readmissions & Length of stay & A\&E use & Costs \\
\hline \multicolumn{6}{|l|}{ Case management } \\
\hline Hickam et al (2013) & $?$ & & & & $?$ \\
\hline Hutt et al (2004) & $?$ & & $?$ & $?$ & $?$ \\
\hline Latour et al (2007) 25 & & $?$ & $?$ & $=$ & \\
\hline Manderson et al (2012) & & & & & - \\
\hline Oeseburg et al (2009) & $?$ & & $?$ & $?$ & $?$ \\
\hline Stokes et al (2015) & & & & & $=$ \\
\hline Taylor et al (2005) ${ }^{44}$ & & $?$ & & & \\
\hline Thomas et al (2013) ${ }^{45}$ & - & & & & \\
\hline \multicolumn{6}{|l|}{ Chronic care model } \\
\hline Adams et al (2007) ${ }^{46}$ & - & & - & - & - \\
\hline de Bruin et al $(2012)^{28}$ & $?$ & & & & - \\
\hline Gonseth et al (2004) $)^{47}$ & & - & & & - \\
\hline Hisashige $(2013)^{69}$ & - & & & & $?$ \\
\hline Kruis et al (2013) & - & & - & & \\
\hline Lemmens et al (2009) ${ }^{49}$ & & $?$ & & & \\
\hline Peytremann-Bridevaux et al (2008) $)^{50}$ & - & & & & \\
\hline Steuten et al $(2009)^{29}$ & & $?$ & & & $=$ \\
\hline Woltmann et al (2012) ${ }^{51}$ & & & & & $=$ \\
\hline \multicolumn{6}{|l|}{ Discharge management } \\
\hline Bettger et al (2012) & & $?$ & & & \\
\hline Brady et al (2005) ${ }^{31}$ & & & & & $?$ \\
\hline Fearon et al (2012) $)^{52}$ & & $=$ & - & & $?$ \\
\hline Feltner et al $(2014)^{53}$ & & - & & & \\
\hline Jeppesen et al (2012) $)^{54}$ & & - & & & - \\
\hline Lambrinou et al (2012) & & - & & & \\
\hline Langhorne et al (2005) & & $=$ & - & & $?$ \\
\hline McMartin $(2013)^{57}$ & & - & - & & \\
\hline Olson et al (2011) & & $=$ & & & \\
\hline Phillips et al (2004) & & - & $=$ & & - \\
\hline Phillips et al (2005) $)^{59}$ & & - & $=$ & & $=$ \\
\hline Prieto-Centurion et al (2014) & & $?$ & & & \\
\hline Tummers et al (2012) & & & & & $?$ \\
\hline Winkel et al (2008) ${ }^{35^{\prime}}$ & & $=$ & & & - \\
\hline Yu et al (2006) ${ }^{36}$ & & $?$ & & & - \\
\hline \multicolumn{6}{|l|}{ Complex interventions } \\
\hline Dickens et al (2014) ${ }^{60}$ & & & & - & \\
\hline Martinez-Gonzelez et al $(2014)^{70}$ & - & - & - & - & $?$ \\
\hline Takeda et al (2012) & & - & & & \\
\hline \multicolumn{6}{|l|}{ Multidisciplinary teams } \\
\hline Health Quality Ontario (2012) $)^{71}$ & - & & & & \\
\hline Health Quality Ontario (2013) ${ }^{37}$ & - & & & & \\
\hline Holland et al (2005) ${ }^{62}$ & - & & - & & - \\
\hline Koshman et al (2008) ${ }^{63}$ & & $?$ & & - & \\
\hline McAlister et al (2004) & $?$ & & & & \\
\hline Medical Advisory Secretariat (2009) 65 & $=$ & & - & & \\
\hline Roccaforte et al (2005) 66 & & - & - & & \\
\hline Sikich $(2012)^{38}$ & - & & & $?$ & \\
\hline Smith et al $(2007)^{39}$ & $?$ & & & & $?$ \\
\hline${ }^{\star}$ Smith et al (2012) ${ }^{40}$ & $?$ & & & & $?$ \\
\hline \multicolumn{6}{|l|}{ Self-management } \\
\hline Franek $(2013)^{41}$ & $=$ & & $=$ & $=$ & \\
\hline Harrison et al (2015) & & $=$ & & & \\
\hline Jovicic et al (2006) ${ }^{42}$ & & - & & & - \\
\hline Smith et al (2012) & $?$ & & & & $?$ \\
\hline Zwerink et al (2014) ${ }^{68}$ & - & & $=$ & & \\
\hline
\end{tabular}


COPD. ${ }^{3846485068}$ The most effective interventions were based on the CCM, for which $4 / 5$ reviews showed statistically significant reductions in admission rates following the intervention. Multiple component strategies were associated with reductions of between $22 \%{ }^{46}$ and $32 \%{ }^{48}$ in admission rates for patients with COPD.

MDT interventions were also effective, with $4 / 8$ reviews showing significant reductions in admissions. Effect sizes ranged from $25 \%$ for a COPD MDT with formal links to primary care, ${ }^{38}$ through $26 \%$ for teams that included specialist heart failure expertise, $^{64}$ to $31 \%$ for teams that included pharmacists as collaborators. ${ }^{63}$ One review of structured self-management interventions demonstrated a $43 \%$ reduction in the relative risk of COPD-related admission. ${ }^{68}$ Case management interventions were largely ineffective in reducing admission rates, with $3 / 4$ showing mixed findings, ${ }^{23} 2427$ although one case management intervention for heart failure comprising intensive follow-up that gradually reduced in intensity over time showed a potential $58 \%$ reduction in admissions. ${ }^{45}$

Most reviews reported condition-specific admissions and admissions for any cause. In all cases, potential reductions in condition-specific admissions were substantially greater than those for all-cause admissions. ${ }^{38} 4562-6468$

\section{Hospital readmissions}

Twenty-four reviews assessed readmissions. Eleven reported positive findings: eight for heart failure, $^{42} 47535558596166$ two for chronic disease ${ }^{57} 70$ and one for COPD. ${ }^{54}$ Discharge management was the most effective intervention, with 6/13 reviews showing significant reductions in readmission rates. ${ }^{53-55} 57-59$ Interventions incorporating an inpatient phase and postdischarge support at home were associated with reductions in condition-specific readmission rates of $24 \%{ }^{32}$ and $49 \%{ }^{53}$ for heart failure interventions, $24 \%$ for a hospital at home intervention for $\mathrm{COPD}^{54}$ and a $15 \%$ reduction for patients with chronic diseases. ${ }^{57}$ Similarly, 'complex' interventions that included specialist nurse-led clinics for heart failure follow-up were associated with a $91 \%$ reduction in condition-specific readmission rates in one review, ${ }^{59}$ and postdischarge hospital outreach coordinated by a MDT was associated with a $32 \%$ reduction in heart failure readmission rates. ${ }^{55}$ In contrast, discharge interventions for patients who had stroke were ineffective, with $0 / 4$ reviews assessing this intervention showing no differences between intervention and control groups. ${ }^{32} 355256$

Other interventions showed less comprehensive evidence. One of three CCM reviews that assessed readmissions found a $30 \%$ reduction in readmission rates for heart failure. ${ }^{47}$ One self-management review in which nurses provided heart failure-specific education reported a $56 \%$ reduction in readmissions. ${ }^{42}$ Two reviews assessing complex interventions reported significant reductions in readmission rates: one for a heart failure case management intervention ${ }^{61}$ and another for patients with general chronic diseases. ${ }^{70}$ One MDT review showed a
$42 \%$ reduction in heart-failure specific readmission, with subgroup analysis indicating that heart failure specialist nurses could reduce condition-specific readmissions by up to $39 \%$. $^{66}$

As with admissions, potential reductions in readmissions were substantially greater for condition-specific readmissions than all-cause readmissions, with effect sizes in the former typically double those for the latter. $^{47535666}$

\section{Length of stay}

Sixteen reviews assessed LoS, across six intervention categories. Neither case management interventions $24 \quad 2527$ or self-management interventions ${ }^{41} 68$ showed evidence of effectiveness, but there were positive findings in the $\mathrm{CCM}^{46}{ }^{48}$ discharge management, ${ }^{52} 5657$ complex intervention $^{60}$ and MDT groups. ${ }^{62} 6566$ Two CCM interventions were associated with a significantly reduced mean LoS for COPD of $2.51^{46}$ and 3.78 days, respectively. ${ }^{48}$

Three discharge management reviews showed significant LoS reductions. Two were for patients who had stroke, including postdischarge support coordinated through multidisciplinary hospital outreach ${ }^{52}$ and early supported discharge. ${ }^{56}$ Pooled results from the early supported discharge meta-analysis suggested a mean LoS reduction of 7.7 days, rising to 28 days for the most severely impaired patients compared to 4 days for moderately impaired patients. ${ }^{56}$ One discharge management intervention for patients with chronic diseases reported positive results, with a modest reduction of 0.91 days. ${ }^{57}$

Finally, three MDT interventions showed significant reductions in LoS, all for heart failure patients. Again, reductions were modest at 1.9 days for an MDT that included a clinician plus specialist nurse, pharmacy, health education, dietician and social worker support, ${ }^{62}$ a 'generally shorter' LoS for an intervention based on nurses, heart failure physicians and general practitioners (GPs) providing condition-specific patient education ${ }^{65}$ and a MDT providing hospital outreach for at least 12 months after hospital discharge was associated with a mean reduction in LoS of 1.49 days. ${ }^{66}$

\section{Accident and emergency use}

Nine reviews measured the effectiveness of interventions in reducing Accident and Emergency (A\&E) use. Five reviews included patients with chronic diseases, all showing mixed findings or no association between intervention and outcome. ${ }^{24} \quad 25 \quad 27 \quad 41 \quad 70$ The remaining reviews assessed single conditions, with $2 / 3$ demonstrating statistically significant reductions in $A \& E$ use for COPD, ${ }^{46} 60$ and one showing significant findings in patients with heart failure. ${ }^{65}$

Case management and self-management interventions were ineffective in reducing A\&E use. ${ }^{24} 252741$ Effective interventions related to the CCM, where multicomponent COPD interventions were associated with a $42 \%$ reduction in A\&E use, ${ }^{46}$ the complex intervention group, where interventions with multiple components 
administered by multiple professionals demonstrated a potential $32 \%$ reduction in A\&E use, ${ }^{60}$ and the MDT group, where one review found a significant reduction in A\&E use when an MDT for heart failure contained condition-specific specialist expertise. ${ }^{65}$ However, A\&E use remained high overall, with $77 \%$ of patients in the intervention group having at least one emergency department visit during the 12-month follow-up period, compared to $84 \%$ of control patients.

\section{Costs}

Twenty-five reviews assessed healthcare costs but the evidence base was poor and heterogeneous-information on potential cost savings was typically qualitative and could not be compared across reviews. Ten reviews reported positive findings: five for patients with heart failure, ${ }^{3642475864}$ two for COPD, ${ }^{46}{ }^{54}$ two for chronic disease $^{26} 28$ and one for stroke. ${ }^{35}$ Eleven reviews reported mixed findings, all for chronic

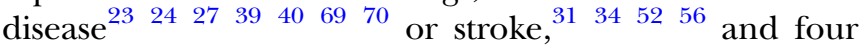
reported no difference in costs between intervention and control groups for chronic disease, ${ }^{43}$ COPD, ${ }^{28}$ mental health ${ }^{51}$ or heart failure. ${ }^{59}$

The most effective interventions were based on the CCM, with three reviews reporting significantly reduced costs. $^{28} 4647$ One review reported cost savings of between $34 \%$ and $70 \%$ for CCM interventions but gave no further detail of the nature of these savings. ${ }^{46}$ Discharge management interventions were cost-effective in some cases, ${ }^{35} 365458$ predominantly due to reduced hospitalisation costs and fewer patient bed days. MDT interventions that included specialist expertise also showed some evidence for cost-effectiveness but again, little detail was given to substantiate this. ${ }^{64}$

\section{DISCUSSION}

The primary aim of this review was to assess whether integrated care-through interventions to coordinate care across two or more health and/or social care settings for patients with chronic diseases-can reduce hospital activity and if so, to what extent. Despite the diverse evidence base and variations within and across reviews in terms of the characteristics, duration and intensity of interventions, some positive trends were evident. Overall, the most effective interventions included discharge planning and postdischarge support for hospital inpatients, ${ }^{53-55}$ 57-59 MDT care-particularly when condition-specific specialists, specialist nurses or pharmacists were part of the team skill mix, ${ }^{3863-65}$ and interventions based on multiple components of the $\mathrm{CCM}^{28}{ }^{28}{ }^{46-48} 50 \quad 69$ although no CCM reviews reported which specific components were most likely to produce positive outcomes. Self-management showed most promise when incorporated into MDT care or when tailored patient education was included in discharge planning. ${ }^{42} 68$ The least effective intervention was case management. Although in theory this intervention may increase health service efficiency by reducing unnecessary contacts with healthcare professionals, ${ }^{43}$ we found little evidence of effectiveness. Some of the key features of effective interventions are outlined in table 5. This table is not intended as a 'toolkit' for effectiveness, since interventions or components that reduced hospital activity for some outcomes and/or conditions were not necessarily effective for others. Nevertheless, it summarises some of the 'ingredients' of potentially effective integrated care interventions.

All hospital activity outcomes showed some significant reductions. Proportionally, LoS was the most likely to reduce, with $9 / 16$ reviews reporting positive findings. However, gains were typically modest: multicomponent CCM strategies could reduce LoS by $2.5-4$ days, ${ }^{46} 48$ and MDT care with specialist expertise was associated with LoS reduction of 1.5-2 days. ${ }^{62}{ }^{66}$ For admissions, 11/21 reviews demonstrated positive findings, suggesting potential reductions of between $15 \%$ and $50 \%$. Readmission rates were significantly reduced in 11/24 reviews, suggesting a $10-30 \%$ reduction in all-cause readmission and a $25-50 \%$ reduction in condition-specific readmission could be achieved with interventions based on discharge management, ${ }^{52-55}$ MDT $^{66}$ and the CCM. ${ }^{46} 485069$ A\&E use typically reduced by $30-40 \%$ in reviews of effective interventions. ${ }^{46} 6065$ It has been argued that integrated care may increase hospital activity due to supply induced demand, in which integration uncovers unmet patient need. ${ }^{72} 73$ Several reviews noted minor increases in activity following case management, ${ }^{24}{ }^{27} \mathrm{CCM},{ }^{51}$ discharge management ${ }^{31}$ and MDT interventions. ${ }^{39} 65$ However, these increases were typically restricted to one or two primary studies within a review and were rarely statistically significant.

A secondary objective was to assess the settings and patient populations for which promising interventions may be most effective. Interventions focused on single conditions showed greater effectiveness than those implemented for patients with general chronic diseases. Those that assessed MDT care or discharge management for patients with heart failure and COPD were typically effective in reducing admissions, ${ }^{38} 62-64$ readmissions ${ }^{53-55} 5859$ and $\operatorname{LoS},{ }^{62} 6566$ with some positive trends evident in reducing A\&E use. ${ }^{65}$ This may reflect the difficulty of designing effective interventions for people with a broad range of conditions, in a healthcare system where care for patients with complex needs remains largely centred on single condition guidelines. Furthermore, interventions such as MDT have been an established feature of disease management for conditions like heart failure for a number of years, and the particular success of interventions focused on this patient group is likely to reflect this. Care offered in patients' homes, whether following discharge from hospital, ${ }^{53}{ }^{54}$ through MDT care,${ }^{386264}$ or through self-management interventions ${ }^{42} 68$ was significantly associated with reduced hospital activity, particularly when home care was coordinated by 
Table 5 Summary of intervention effectiveness

Intervention/feature*
Complex interventions with multiple components
$\begin{aligned} & \text { Postdischarge hospital outreach coordinated by a } \\ & \text { multidisciplinary team }\end{aligned}$

multidisciplinary team

\section{MDT with:}
A. Disease-specific specialists as core members
B. Specialist nurse-led clinics
C. Pharmacists as collaborative partners

Transition from hospital to home is most effective when interventions are initiated during the inpatient phase and continue postdischarge

Home-based community follow-up

Self-management education combined with multidisciplinary approaches or discharge planning

\section{Notes/caveats}

- Greatest effects when treating patients with single rather than multiple conditions

- No reviews stated specific components that were more (or less) likely to be effective than others

- Greatest effects when treating patients with single rather than multiple conditions

- In contrast, community inreach interventions not effective, even when featuring MDT

- Not effective for patients who had stroke

- Greatest effects when treating patients for single conditions

- No reviews compared the 'added value' that a given professional or clinician may bring to a MDT, so optimal composition remains unknown

- Effective for reducing length of stay in patients who had stroke

- Community follow-up least likely to be effective when delivered through a case management model

- Greatest effects when treating patients for single conditions

- Simple self-management interventions were largely ineffective

${ }^{*} \mathrm{MDT}$, multidisciplinary team

multidisciplinary outreach as opposed to a community in-reach model. Although these interventions were associated with significantly reduced hospital activity, the most successful were coordinated by the acute sector, suggesting that effective integrated care may still rely on the deployment of substantial hospital resources and the involvement of multiple acute sector healthcare professionals.

Our final objective was to assess the cost implications of integrated care interventions. Data were poor: the care components that cost data referred to were often unclear and effect sizes were rarely stated. Where statistical significance was described, the majority of savings appeared to come from a reduction in costs incurred through hospitalisation, whether this was because interventions allowed patients to be discharged from hospital earlier or whether interventions reduced subsequent rates of hospitalisation or rehospitalisation. As a result, interventions which included some element of home care or rehabilitation tended to be cost saving compared to care in which rehabilitation was provided

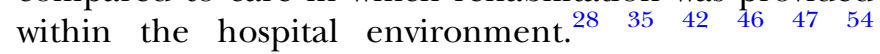
However, it is likely that substantial cost savings can only be realised if hospital capacity can be physically removed from the system, for example, through ward closures. We found little evidence of this following integrated care interventions.

\section{Strengths and weaknesses}

This is the first umbrella review of its kind and is timely given the increasing emphasis on integrated care in healthcare policy with the key aim of reducing hospital use. By undertaking an umbrella review of systematic reviews, we could assess a large volume of evidence across diverse conditions, interventions and outcomes. However, umbrella reviews have limitations. Grouping interventions in a way that allowed meaningful conclusions to be drawn about their effectiveness was challenging. Although we employed the Effective Practice and Organisation of Care Group (EPOC) taxonomy ${ }^{74}$ as an organisational framework, few interventions were mutually exclusive and the characteristics and form of interventions frequently overlapped. For example, most discharge management interventions were delivered by MDT, and several interventions included some element of self-management support. Even for reviews which shared broadly similar intervention characteristics, the duration of follow-up, study design, complexity, intensity and mode of delivery varied. Furthermore, because the unit of analysis is the review rather than the primary study level, the re-synthesis of information at the umbrella review level that has already been synthesised at review level risks loss of detail or misinterpretation of findings and trends. But, by restricting inclusion to reviews receiving moderate, good or high QA scores, we 
reduced the likelihood of evidence misinterpretation and the incidence of discordant findings. Successful approaches to integrated care have highly contextspecific histories, yet by undertaking an umbrella review, we were unable to draw conclusions about the specific contexts in which interventions were implemented. Nevertheless, we believe that the methodological strengths of our approach outweigh the limitation of being unable to comment on the specific contexts in which interventions were implemented.

\section{Implications for clinicians and policymakers}

Although there was evidence that some integrated care interventions can reduce hospital activity, effects were rarely unequivocally positive. The size of gains from integration may also be modest. ${ }^{17}$ For example, in recent years, the trend in outcomes such as length of hospital stay has been steadily reducing, largely due to improved surgical techniques and increased day case treatment. ${ }^{8}$ This suggests that there may be limits to the absolute reductions in key hospital activity metrics that integrated care initiatives could achieve. ${ }^{1}$ This was evident in several reviews that noted statistically significant differences in outcomes for intervention versus control patients, but which reported persistently high absolute rates of outcomes such as admissions and readmissions in each group. ${ }^{485254586568}$

This has implications for the potential success of policy initiatives designed to reduce hospital activity. In England, integration has become a central feature of the evolving healthcare policy landscape and there are high expectations of substantial benefits from integrating care. The BCF and 'Vanguard' sites ${ }^{7}$ have been developed following recognition that radically different models of care are needed if the NHS in England is to overcome its growing challenges, and both policy initiatives involve farreaching change to health and social care services with the aim of meeting national headline targets for reduced hospital and emergency care use. ${ }^{75}$ Interventions shown to be effective in this review have much in common with the rationale behind the $\mathrm{BCF}$ - care provided in the community rather than in hospitals was shown in many cases to be highly effective. Multidisciplinary care, discharge planning and self-management educating patients on identifying symptoms of exacerbation of their condition (s) all have the potential to improve outcomes and reduce activity at the 'back door' and 'front door' of the acute sector. Disease-specific expertise was also found in many reviews to be crucial to the success of integrated care interventions, as was secondary care outreach to other settings. This bodes well for BCF and Vanguard initiatives built around these interventions. However, it is of concern that many vanguard sites aim to integrate care via a case management approach, which showed the poorest evidence of effectiveness in our review. This raises questions over whether the Vanguard strategies will be able to deliver the outcome improvements they are being established to achieve. The extent to which integrated care can bring about significant cost savings in a health system beset with ongoing budgetary constraints is also highly uncertain.

Interventions designed for single conditions were substantially more effective than those designed to treat patients with chronic diseases in general terms. On one hand, this suggests that service providers can achieve some 'quick wins' by targeting interventions such as discharge planning and specialist MDT towards specific patient groups in whom the evidence for reduced hospital use is clear. On the other hand, this means that integration may not deliver the substantial reductions in acute sector activity that must be achieved if healthcare services are to remain sustainable in the longer term.

\section{Unanswered questions and future research}

Integrated care poses challenges to the measurement of 'hard' healthcare service outcomes in what are often complex intervention programmes. Determining cause and effect is difficult when interventions include multiple components, yet being able to link a specific intervention to a particular observed outcome is typically central to policymaking and commissioning objectives. Research to develop a robust taxonomy for integrated care interventions and their components would make assessments of comparative effectiveness across interventions less challenging. We attempted to maximise the relevance of review findings to the English health and social care system by considering interventions implemented in developed economies, but further research is needed to determine whether interventions found to be effective in other healthcare systems can be generalised to the NHS. In particular, robust evaluations would allow the influence of local and organisational contexts to be disentangled from the effects of the intervention themselves, as although the umbrella review gives some indication about 'what' might work, it does not necessarily help our understanding of 'how' an intervention works and why it may work in some circumstances and not others.

Few reviews explicitly addressed multimorbidity, which has recently become of central importance in debates about hospital use by patients with complex needs. ${ }^{4} 76$ Further research is needed to understand the issues faced by patients with multimorbidity when negotiating the health and social care system. ${ }^{77}$ Similarly, despite our comprehensive search strategy, the evidence base focused little on the role of primary care, social care or the voluntary sector in providing integrated services. Given current policy drivers towards services being provided in the community by GPs and other organisations rather than acute providers, further research to assess the implications of integrated care for the organisation and delivery of services in these sectors is urgently needed.

\section{CONCLUSIONS}

This review highlights a number of potentially effective integrated care interventions to reduce hospital use for 
patients with chronic diseases. Interventions based on MDT that include condition specialists, those focused on discharge management that include postdischarge rehabilitation and follow-up and those based on multicomponent strategies were most likely to be associated with significant reductions in hospital use for patients with single conditions such as heart failure and COPD. Yet there was little robust evidence about potential cost efficiencies, and the effectiveness of care delivered in primary and social care settings remains largely unknown. Despite considerable fanfare accompanying efforts to integrate care across the health and social care system in England, integration does not seem to be a 'magic bullet' and the magnitude of achievable gains is unlikely to match those required by current policy targets.

Acknowledgements The authors thank Sue Bayliss for performing literature searches; Magdalena Skrybant (PPI representative) for her valuable comments at the study design stage; Professor Jon Glasby for his input to the protocol and Professor Christian Mallen for his comments on the draft of this paper.

Contributors SD, GC and SF designed the study and the literature search strategy. SD and SF undertook data cleaning, title and abstract screening, full paper assessment, data extraction and analysis of all data, with input from GC as needed. SD drafted and revised the paper and is guarantor for the work. SF and GC critically revised the paper for intellectual content. All authors gave final approval of the manuscript and are accountable for all aspects of the accuracy and integrity of the work.

Funding This research was funded by the National Institute of Health Research (NIHR) Collaboration for Leadership in Applied Health Research and Care West Midlands (CLAHRCWM).

Disclaimer The study sponsor and funder had no role in the study design, in the collection, analysis and interpretation of data, in the writing of the report and in the decision to submit the article for publication.

This paper presents independent research funded by the National Institute of Health Research (NIHR) Collaboration for Leadership in Applied Health Research and Care West Midlands (CLAHRCWM). The views expressed are those of the authors and not necessarily those of the NHS, the NIHR or the Department of Health.

\section{Competing interests None declared.}

Provenance and peer review Not commissioned; externally peer reviewed.

Data sharing statement No additional data are available.

Data access All authors had full access to all of the data (including statistical reports and tables) in the study and can take responsibility for the integrity of the data and the accuracy of the data analysis.

Open Access This is an Open Access article distributed in accordance with the terms of the Creative Commons Attribution (CC BY 4.0) license, which permits others to distribute, remix, adapt and build upon this work, for commercial use, provided the original work is properly cited. See: http:// creativecommons.org/licenses/by/4.0/

\section{REFERENCES}

1. Smith $\mathrm{P}, \mathrm{McKeon} \mathrm{A}$, Blunt I, et al. NHS hospitals under pressure: trends in acute activity up to 2022. London: Nuffield Trust, 2014.

2. Ham C, Dixon A, Brooke B. Transforming the delivery of health and social care: the case for fundamental change. London: The Kings Fund, 2012.

3. Department of Health. Long term conditions compendium of information. 3rd edn. London: Department of Health, 2012.

4. Fortin $\mathrm{M}$, Soubhi $\mathrm{H}$, Hudon $\mathrm{C}$, et al. Multimorbidity's many challenges. BMJ 2007;334:1016-7.
5. Shaw $\mathrm{S}$, Rosen $\mathrm{R}$, Rumbold $\mathrm{B}$. What is integrated care? London: Nuffield Trust, 2011.

6. Glasby J, Dickinson H. Partnership working in health and social care: what is integrated care and how can we deliver it? 2nd edn Bristol: Policy Press, 2013.

7. NHS England, Care Quality Commission, Health Education England Monitor, Public Health England, Trust Development Authority. Five year forward view. London: NHS England, 2014.

8. Alderwick H, Ham C, Buck D. Population health systems: going beyond integrated care. London: The King's Fund, 2015.

9. National Voices. What patients want from integration; 2012. http:// www.nationalvoices.org.uk/sites/www.nationalvoices.org.uk/files/ what_patients_want_from_integration_national_voices_paper.pdf

10. Mastellos N, Gunn L, Harris M, et al. Assessing patients' expectations of integrated care: a survey of patient views from the North West London integrated care pilot. Int $J$ Integr Care 2014;14:1-9.

11. Mathers N, Patel V, Thomas M. General practice and the integration of care: an RCGP policy report. London: Royal College of General Practitioners, 2012

12. Goodwin N, Smith J, Davies A, et al. Integrated care for patients and populations: improving outcomes by working together: a report to the Department of Health and NHS Future Forum. London: The Kings Fund, 2012.

13. Ouwens $\mathrm{M}$, Wollersheim $\mathrm{H}$, Hermens $\mathrm{R}$, et al. Integrated care programmes for chronically ill patients: a review of systematic reviews. Int J Qual Health Care 2005;17:141-6.

14. Rosen R. International case studies of integrated care. London: Nuffield Trust, 2010.

15. Goodwin N, Sonola L, Thiel V, et al. Co-ordinated care for people with complex chronic conditions: key lessons and markers for success. London: The Kings Fund, 2013.

16. Wistow $\mathrm{G}$, Gaskins $\mathrm{M}$, Holder $\mathrm{H}$, et al. Putting integrated care into practice: the North West London experience. London: Nuffield Trust, 2015.

17. Bardsley M, Steventon A, Smith J, et al. Evaluating integrated and community-based care. London: Nuffield Trust, 2013.

18. Pieper D, Antoine SL, Mathes T, et al. Systematic review finds overlapping reviews were not mentioned in every other overview. J Clin Epidemiol 2014;67:368-75.

19. Damery S, Flanagan S, Combes G. The effectiveness of interventions to achieve co-ordinated multidisciplinary care and reduce hospital use for people with chronic diseases: study protocol for a systematic review of reviews. Syst Rev 2015;4:64.

20. Barnett K, Mercer SW, Norbury M, et al. Epidemiology of multimorbidity and implications for health care, research and medica education: cross-sectional study. Lancet 2012;380:37-43.

21. Diederichs $\mathrm{C}$, Berger K, Bartels DB. The measurement of multiple chronic diseases - a systematic review on existing multimorbidity indices. J Gerontol A Biol Sci Med Sci 2011;66:301-11.

22. Health and Social Care Information Centre. Health survey for England. London: HSCIC, 2013.

23. Hickam DH, Weiss JW, Guise JM, et al. Outpatient case management for adults with medical illness and complex care needs. Rockville (MA): Agency for Healthcare Research and Quality, 2013.

24. Hutt R, Rosen R, McCauley J. Case-managing long term conditions: what impact does it have in the treatment of older people? London: The Kings Fund, 2004

25. Latour CHM, van der Windt DAWM, de Jonge $P$, et al. Nurse-led case management for ambulatory complex patients in general health care: a systematic review. J Psychosom Res 2007;62:385-95.

26. Manderson B, Mcmurray J, Piraino E, et al. Navigation roles support chronically ill older adults through healthcare transitions: a systematic review of the literature. Health Soc Care Community 2012;20:113-27.

27. Oeseburg B, Wynia K, Middel B, et al. Effects of case management for frail older people or those with chronic illness: a systematic review. J Nurs Res 2009;58:201-10.

28. de Bruin SR, Versnel N, Lemmens LC, et al. Comprehensive care programs for patients with multiple chronic conditions: a systematic literature review. Health Policy 2012;107:108-45.

29. Steuten LMG, Lemmens KMM, Nieboer AP, et al. Identifying potentially cost effective chronic care programs for people with COPD. Int J COPD 2009;4:87-100.

30. Prvu Bettger J, Alexander KP, Dolor RJ, et al. Transitional care after hospitalization for acute stroke or myocardial infarction. Ann Intern Med 2012;157:407-16.

31. Brady BK, McGahan L, Skidmore B. Systematic review of economic evidence on stroke rehabilitation services. Int J Technol Assess Health Care 2005;21:15-21. 
32. Olson DM, Bettger J, Alexander KP, et al. Transition of care for acute stroke and myocardial infarction patients: from hospitalization to rehabilitation, recovery and secondary prevention. Evidence report no 202, 2011; AHRQ publication 11(12)-E011. Agency for Healthcare Research and Quality, October 2011.

33. Prieto-Centurion V, Markos MA, Ramey NI, et al. Interventions to reduce hospitalizations after chronic obstructive pulmonary disease exacerbations: a systematic review. Ann Am Thorac Soc 2014;11:417-24.

34. Tummers JF, Schrijvers AJ, Visser-Meily JM. Economic evidence on integrated care for stroke patients: a systematic review. Int $J$ Int Care 2012;12:e193.

35. Winkel A, Ekdahl C, Gard G. Early discharge to therapy-based rehabilitation at home in patients with stroke: a systematic review. Phys Ther Rev 2008:13:167-87.

36. Yu DSF, Thompson DR, Lee DTF. Disease management programmes for older people with heart failure: crucial characteristics which improve post-discharge outcomes. Eur Heart $J$ 2006;27:596-612.

37. Health Quality Ontario. Continuity of care to optimize chronic disease management in the community setting: an evidence-based analysis. Ont Health Technol Assess Ser 2013;13:1-41.

38. Sikich N. Community-based multidisciplinary care for patients with stable chronic obstructive pulmonary disease (COPD): an evidence-based analysis. Ont Health Technol Assess Ser 2012;12:1-51.

39. Smith SM, Allwright S, O'Dowd T. Effectiveness of shared care across the interface between primary and speciality care in chronic disease management. Cochrane Database Syst Rev 2007;(3):CD004910.

40. Smith SM, Soubhi H, Fortin M, et al. Interventions for improving outcomes in patients with multimorbidity in primary care and community settings. Cochrane Database Syst Rev 2012;(4): CD006560.

41. Franek J. Self-management support interventions for persons with chronic disease: an evidence-based analysis. Ont Health Technol Assess Ser 2013;13:1-60.

42. Jovicic A, Holroyd-Leduc JM, Straus SE. Effects of self-management intervention on health outcomes of patients with heart failure: a systematic review of randomized controlled trials. BMC Cardiovasc Disord 2006;6:43.

43. Stokes J, Panagioti M, Alam R, et al. Effectiveness of case management for 'at risk' patients in primary care: a systematic review and meta-analysis. PLOS ONE 2015;10:e0132340.

44. Taylor SJ, Candy B, Bryar RM et al. Effectiveness of innovations in nurse led chronic disease management for patients with chronic obstructive pulmonary disease: systematic review of evidence. BMJ 2005;331:485.

45. Thomas R, Huntley A, Mann M, et al. Specialist clinics for reducing emergency admissions in patients with heart failure: a systematic review and meta-analysis of randomised controlled trials. Heart 2013;99:233-9.

46. Adams SG, Smith PK, Allan PF, et al. Systematic review of the chronic care model in chronic obstructive pulmonary disease prevention and management. Arch Intern Med 2007;167:551-61.

47. Gonseth J, Guallar-Castillón P, Banegas JR, et al. The effectiveness of disease management programmes in reducing hospital re-admission in older patients with heart failure: a systematic review and meta-analysis of published reports. Eur Heart $J$ 2004;25:1570-95.

48. Kruis AL, Smidt N, Assendelft WJ, et al. Integrated disease management interventions for patients with chronic obstructive pulmonary disease. Cochrane Database of Syst Rev 2013;(10):CD009437.

49. Lemmens KM, Nieboer AP, Huijsman R. A systematic review of integrated use of disease-management interventions in asthma and COPD. Resp Med 2009;103:670-91.

50. Peytremann-Bridevaux I, Staeger P, Bridevaux PO, et al. Effectiveness of chronic obstructive pulmonary disease management programmes: systematic review and meta-analysis. Am J Med 2008:121:433-43.

51. Woltmann E, Grogan-Kaylor A, Perron B, et al. Comparative effectiveness of collaborative chronic care models for mental health conditions across primary, specialty and behavioural health care settings: systematic review and meta-analysis. Am J Psychiatry 2012;169:790-804.

52. Fearon P, Langhorne P, Early Supported Discharge Trialists. Services for reducing duration of hospital care for acute stroke patients (review). Cochrane Database Syst Rev 2012;(9):CD000443.
53. Feltner $\mathrm{C}$, Jones $\mathrm{CD}$, Cené $\mathrm{CW}$, et al. Transitional care interventions to prevent readmissions for persons with heart failure: a systematic review and meta-analysis. Ann Intern Med 2014;160:774-84.

54. Jeppesen E, Brurberg KG, Vist GE, et al. Hospital at home for acute exacerbations of chronic obstructive pulmonary disease. Cochrane Database Syst Rev 2012;(5):CD003573.

55. Lambrinou E, Kalogirou F, Lamnisos D, et al. Effectiveness of heart failure management programmes with nurse-led discharge planning in reducing re-admissions: a systematic review and meta-analysis. Int J Nurs Stud 2012;49:610-24.

56. Langhorne P, Taylor G, Murray G, et al. Early supported discharge services for stroke patients: a meta-analysis of individual patients' data. Lancet 2005;365:501-6.

57. McMartin K. Discharge planning in chronic conditions: an evidence based analysis. Ont Health Technol Assess Ser 2013;13:1-72.

58. Phillips CO, Wright SM, Kern DE, et al. Comprehensive discharge planning with postdischarge support for older patients with congestive heart failure: a meta analysis. JAMA 2004;291:1358-67.

59. Phillips $\mathrm{CO}$, Singa RM, Rubin HR, et al. Complexity of program and clinical outcomes of heart failure disease management incorporating specialist nurse-led heart failure clinics: a meta-regression analysis. Eur J Heart Fail 2005;7:333-41.

60. Dickens $\mathrm{C}$, Katon W, Blakemore A, et al. Complex interventions that reduce urgent care use in COPD: a systematic review with meta-regression. Resp Med 2014;108:426-37.

61. Takeda A, Taylor SJ, Taylor RS, et al. Clinical service organisation for heart failure. Cochrane Database Syst Rev 2012;(9):CD002752.

62. Holland R, Battersby J, Harvey I, et al. Systematic review of multidisciplinary interventions in heart failure. Heart 2005;91:899-906

63. Koshman SL, Charrois TL, Simpson SH, et al. Pharmacist care of patients with heart failure: a systematic review of randomized trials. Arch Intern Med 2008;168:687-94.

64. McAlister FA, Stewart S, Ferrua S, et al. Multidisciplinary strategies for the management of heart failure patients at high risk for admission: a systematic review of randomised trials. J Am Coll Cardiol 2004:44:810-19.

65. Medical Advisory Secretariat. Community-based care for the specialized management of heart failure. Ont Health Technol Assess Ser 2009:9:1-42.

66. Roccaforte R, Demers C, Baldassarre F, et al. Effectiveness of comprehensive disease management programmes in improving clinical outcomes in heart failure patients. A meta-analysis. Eur J Heart Fail 2005;7:1133-44.

67. Harrison SL, Janaudis-Ferreira T, Brooks D, et al. Self-management following an acute exacerbation of COPD: a systematic review. Chest 2015;147:646-61.

68. Zwerink M, Brusse-Keizer M, van der Valk PD, et al. Self management for patients with chronic obstructive pulmonary disease (review). Cochrane Database Syst Rev 2014;(3):CD002990.

69. Hisashige A. The effectiveness and efficiency of disease management programs for patients with chronic diseases. Glob $J$ Health Sci 2013;5:27-48.

70. Martinez-González NA, Berchtold P, Ullman K, et al. Integrated care programmes for adults with chronic conditions: a meta-review. Int J Qual Health Care 2014:26:561-70.

71. Health Quality Ontario. Specialized community-based care: an evidence-based analysis. Ont Health Technol Assess Ser 2012;12:1-60.

72. Roland M, Lewis R, Steventon A, et al. Case management for at-risk elderly patients in the English integrated care pilots: observational study of staff and patient experience and secondary care utilisation. Int $J$ Int Care 2012;12:1-21.

73. Steventon A, Bardsley M, Billings J, et al. An evaluation of the impact of community-based interventions on hospital use. London: Nuffield Trust, 2011.

74. Cochrane Effective Practice and Organisation of Care Group (EPOC). EPOC taxonomy. Oslo: Norwegian Knowledge Centre for the Health Services, 2002.

75. Naylor $\mathrm{C}$, Alderwick $\mathrm{H}$, Honeyman M. Acute hospitals and integrated care: from hospitals to health systems. London: Kings Fund, 2015.

76. Bower P. Better management of multimorbidity: a critical look at the Ariadne principles. BMC Med 2014;12:222.

77. Paddison CA, Saunders CL, Abel GA, et al. Why do patients with multimorbidity in England report worse experiences in primary care? Evidence from the General Practice Patient Survey. BMJ Open 2015:5:e006172. 